\title{
Josef Král
}

Flows of heat and the Fourier problem

Czechoslovak Mathematical Journal, Vol. 20 (1970), No. 4, 556-598

Persistent URL: http://dml.cz/dmlcz/100984

\section{Terms of use:}

(C) Institute of Mathematics AS CR, 1970

Institute of Mathematics of the Czech Academy of Sciences provides access to digitized documents strictly for personal use. Each copy of any part of this document must contain these Terms of use.

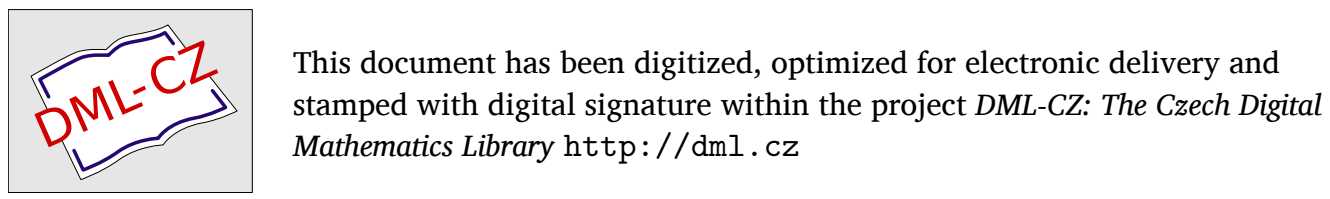




\title{
FLOWS OF HEAT AND THE FOURIER PROBLEM
}

\author{
Josef KráL, Praha
}

(Received June 19, 1969)

\section{INTRODUCTION}

Let $D$ be an arbitrary open set in $R^{m}$, the Euclidean $m$-space, and suppose that its boundary $B$ is compact and non-void. Fix $T_{1}, T_{2} \in R^{1}, T_{1}<T_{2}$, and let

$$
C=B \times\left\langle T_{1}, T_{2}\right), \quad E=D \times\left(T_{1}, T_{2}\right) .
$$

By the term measure we shall usually mean a finite signed Borel measure in some Euclidean space. If $\mu$ is a measure and $M$ is a Borel set in the domain of $\mu$, then $|\mu|(M)$ will denote the variation of $\mu$ on $M$. Let $\mathscr{B}^{\prime}\left(T_{1}, T_{2}\right)=\mathscr{B}^{\prime}$ stand for the class of all measures $\mu$ in $R^{m+1}$ with

$$
|\mu|\left(R^{m+1} \backslash C\right)=0 .
$$

With each $\mu \in \mathscr{B}^{\prime}$ we associate the corresponding thermal potential

$$
U \mu(z)=\int G(z-\zeta) \mathrm{d} \mu(\zeta), \quad z \in E,
$$

where $G(z)=0$ for $z=\left[z_{1}, \ldots, z_{m+1}\right]$ with $z_{m+1} \leqq 0$, while for $z_{m+1}>0$

$$
G(z)=z_{m+1}^{-m / 2} \exp \left(-\sum_{j=1}^{m} z_{j}^{2} / 4 z_{m+1}\right) \text {. }
$$

Writing $\partial_{j}$ for the derivative with respect to the $j$-th variable one easily verifies that $\partial_{j} U \mu$ are integrable over $E$ for $j=1, \ldots, m$. This makes it possible to introduce the functional $H \mu$ over the class $\mathscr{D}_{T_{2}}$ of all infinitely differentiable functions with compact support in $R^{m+1} \cap\left\{z ; z_{m+1}<T_{2}\right\}$ defining

$$
\langle\varphi, H \mu\rangle=\int_{E}\left\{\left[\sum_{j=1}^{m} \partial_{j} U \mu(z) \cdot \partial_{j} \varphi(z)\right]-U \mu(z) . \partial_{m+1} \varphi(z)\right\} \mathrm{d} z .
$$


$H \mu$ will be termed the heat flow associated with $\mu$. The reason for the terminology lies in the fact that, in the special case when the boundary $B$ of $D$ is a smooth hypersurface in $R^{m}$ with the exterior normal $n(x)=\left[n_{1}(x), \ldots, n_{m}(x)\right]$ and the derivatives $\partial_{j} U \mu$ extend from $E$ to continuous functions $u_{j}$ on the closure of $E,\langle\varphi, H \mu\rangle$ transforms into

$$
\int_{T_{1}}^{T_{2}}\left\{\int_{B} \varphi(x, t)\left[\sum_{j=1}^{m} u_{j}(x, t) n_{j}(x)\right] \mathrm{d} \sigma_{B}(x)\right\} \mathrm{d} t,
$$

where $\mathrm{d} \sigma_{B}$ is the area element on $B$ (and, of course, $[x, t]$ stands for $\left[x_{1}, \ldots, x_{m}, t\right]$ whenever $x=\left[x_{1}, \ldots, x_{m}\right] \in R^{m}$ and $\left.t \in R^{1}\right)$. In general there is no measure $v_{\mu}$ representing $H \mu$ over $\mathscr{D}_{T_{2}}$. In order to be able to formulate geometric conditions on $D$ guaranteeing the existence of such a $v_{\mu}$ for each $\mu \in \mathscr{B}^{\prime}$ we adopt the following terminology introduced in [10]: Given $x \in R^{m}, r>0$ and $\theta \in \Gamma=R^{m} \cap\{\theta ;|\theta|=1\}$, we call $y \in S_{r}(\theta, x)=\{x+\varrho \theta ; 0<\varrho<r\}$ a hit of $S_{r}(\theta, x)$ on $D$ provided each ball

$$
\Omega_{\varrho}(y)=R^{m} \cap\{v ;|v-y|<\varrho\}
$$

meets both $S_{r}(\theta, x) \backslash D$ and $S_{r}(\theta, x) \cap D$ in a set of positive linear measure. (Note that $\Omega_{\varrho}(y) \cap S_{r}(\theta, x) \cap D$ is open in $S_{r}(\theta, x)$; consequently, it is either void or it has a positive linear measure.) The number (possibly infinite) of all the hits of $S_{r}(\theta, x)$ on $D$ will be denoted by $n_{r}(\theta, x)$. For fixed $r>0$ and $x \in R^{m}, n_{r}(\theta, x)$ is a Baire function of the variable $\theta$ on $\Gamma$ (see [10], proposition 1.6) and one may put

$$
v_{r}(x)=\int_{\Gamma} n_{r}(\theta, x) \mathrm{d} \sigma_{\Gamma}(\theta) .
$$

If $M \neq \emptyset$ is a subset of $B$ we let

$$
V_{0}(M)=\lim _{r \rightarrow 0+} \sup _{x \in M} v_{r}(x)
$$

finally, put $V_{0}(\emptyset)=0$. With this notation we have now the following

Theorem 1. In order that $H \mu$ be representable by means of a measure for each $\mu \in \mathscr{B}^{\prime}$ it is necessary and sufficient that

$$
V_{0}(B)<+\infty
$$

In what follows we always assume (1). For each $\mu \in \mathscr{B}^{\prime}$ there is a uniquely determined measure $v_{\mu}$ satisfying the following conditions (i), (ii):

$$
\begin{gathered}
\varphi \in \mathscr{D}_{T_{2}} \Rightarrow\langle\varphi, H \mu\rangle=\int \varphi \mathrm{d} v_{\mu}, \\
\left|v_{\mu}\right|\left(R^{m} \times\left\langle T_{2},+\infty\right)\right)=0 .
\end{gathered}
$$


It is easily seen that the support of $v_{\mu}$ is contained in $B \times\left\langle T_{1}, T_{2}\right\rangle$; in other words, $v_{\mu} \in \mathscr{B}^{\prime}$ for each $\mu \in \mathscr{B}^{\prime}$. Let us agree to write simply $v_{\mu}=H \mu$ and equip $\mathscr{B}^{\prime}$ with the norm

$$
\|\mu\|=|\mu|\left(R^{m+1}\right)=|\mu|(C) .
$$

Then $H: \mu \rightarrow H \mu$ is a bounded operator on the Banach space $\mathscr{B}^{\prime}$. Let us also quote here that (1) implies

$$
\sup \left\{v_{\infty}(x) ; x \in R^{m}\right\}<+\infty .
$$

Another consequence of (1) is the existence of the density

$$
d_{D}(x)=\lim _{\varrho \rightarrow 0+} \frac{\text { volume }\left(\Omega_{\varrho}(x) \cap D\right)}{\operatorname{volume}\left(\Omega_{\varrho}(x)\right)}
$$

at any $x \in R^{m}$.

Let now $\mathscr{B}\left(T_{1}, T_{2}\right)=\mathscr{B}$ be the Banach space of all continuous functions $f$ on $B \times$ $\times\left\langle T_{1}, T_{2}\right\rangle$ such that $f\left(B \times\left\{T_{2}\right\}\right)=\{0\}$, with the norm

$$
\|f\|=\sup \left\{|f(z)| ; z \in B \times\left\langle T_{1}, T_{2}\right\rangle\right\} .
$$

We shall introduce an operator $W_{0}$ on $\mathscr{B}$ whose dual is $H: W_{0}^{\prime}=H$. For this purpose we recall the following notation introduced in [10]. Given $x \in B$ and $\theta \in \Gamma$ we put for $r>0$

$$
s(r ; x, \theta)=\varepsilon \quad(= \pm 1)
$$

if there is a $\delta>0$ such that

$$
x+(r+\varepsilon \varrho) \theta \in D, \quad x+(r-\varepsilon \varrho) \theta \in R^{m} \backslash D
$$

for almost every $\varrho \in(0, \delta)$; otherwise we set $s(r ; x, \theta)=0$. With each $f \in \mathscr{B}, t \in$ $\epsilon\left\langle T_{1}, T_{2}\right\rangle$ and $\eta>0$ we associate the sum

$$
\sum f\left(x+r \theta, t+\frac{r^{2}}{4 \eta}\right) s(r ; x, \theta)=\sum_{f}([x, t] ; \eta, \theta)
$$

extended over $r \in\left(0,2\left[\eta\left(T_{2}-t\right)\right]^{1 / 2}\right)$ (consequently, $\sum_{f}([x, t] ; \eta, \theta)=0$ if $\left.t=T_{2}\right)$. For fixed $\eta>0$ and $z=[x, t] \in B \times\left\langle T_{1}, T_{2}\right\rangle, \sum_{f}(z ; \eta, \theta)$ is defined almost everywhere and integrable $\mathrm{d} \sigma_{\Gamma}(\theta)$ on $\Gamma$ and the integral

$$
W f(z)=\int_{0}^{\infty} \mathrm{e}^{-\eta} \eta^{(m-1) / 2}\left[\int_{\Gamma} \sum_{f}(z ; \eta, \theta) \mathrm{d} \sigma_{\Gamma}(\theta)\right] \mathrm{d} \eta
$$

is convergent. Writing $\hat{z}=\left[z_{1}, \ldots, z_{m}\right]$ for each $z=\left[z_{1}, \ldots, z_{m}, z_{m+1}\right] \in R^{m+1}$ we are now able to formulate the following 
Theorem 2. For each $f \in \mathscr{B}$ define

$$
W_{0} f(z)=2^{m-1}\left[W f(z)+2 \pi^{m / 2} d_{D}(\hat{z}) f(z)\right], \quad z \in B \times\left\langle T_{1}, T_{2}\right\rangle ;
$$

then $W_{0} f \in \mathscr{B}$. The operator $W_{0}: f \rightarrow W_{0} f$ is bounded on $\mathscr{B}$ and $H$ is dual to $W_{0}$.

Let $I$ stand for the identity operator on $\mathscr{B}$ and consider the operators

$$
W_{\alpha}=W_{0}-2^{m} \pi^{m / 2} \alpha I, \quad \alpha \in R^{1} \backslash\{0\} .
$$

It is useful to evaluate the quantity

$$
\omega W_{\alpha}=\inf \left\|W_{\alpha}-T\right\|,
$$

$T$ ranging over all compact operators acting on $\mathscr{B}$. In particular, in view of the equality

$$
H=\left(2^{m} \pi^{m / 2} \alpha I+W_{\alpha}\right)^{\prime},
$$

it is important to know conditions on $D$ guaranteeing the validity of the following estimate for $g(\alpha)=\omega W_{\alpha} /|\alpha| 2^{m} \pi^{m / 2}$ :

$$
a=\inf \left\{g(\alpha) ; \alpha \in R^{1} \backslash\{0\}\right\}<1 .
$$

They read as follows.

Theorem 3. Let

$$
B_{1}=B \cap\left\{x ; d_{D}(x)=1\right\}, \quad B_{2}=B \cap\left\{x ; d_{D}(x)=\frac{1}{2}\right\}
$$

and write

$$
A=2 \pi^{m / 2} / \Gamma\left(\frac{1}{2} m\right)
$$

for the area of the unit $m$-sphere $\Gamma$. Then (2) holds if and only if

$$
V_{0}\left(B_{1}\right)<A \text { and } V_{0}\left(B_{2}\right)<\frac{1}{2} A \text {. }
$$

If these condition are fulfilled then $\gamma$ yielding

$$
a=g(\gamma)
$$

is uniquely determined and one of the following cases (i)-(iii) must occur:

(i) $B_{1}=\emptyset$,

(ii) $B_{2}=\emptyset$ or $V_{0}\left(B_{1}\right) \geqq V_{0}\left(B_{2}\right)+\frac{1}{2} A$,

(iii) $B_{1} \neq \emptyset \neq B_{2}$ and $\left|V_{0}\left(B_{1}\right)-V_{0}\left(B_{2}\right)\right| \leqq \frac{1}{2} A$. 
The corresponding values of $a$ and $\gamma$ are then given as follows:

(i) $\Rightarrow A a=2 V_{0}\left(B_{2}\right), \gamma=\frac{1}{2}$,

(ii) $\Rightarrow A a=V_{0}\left(B_{1}\right), \gamma=1$,

(iii) $\quad a=\frac{V_{0}\left(B_{1}\right)+V_{0}\left(B_{2}\right)+\frac{1}{2} A}{V_{0}\left(B_{1}\right)-V_{0}\left(B_{2}\right)+\frac{3}{2} A}, \quad \gamma=\frac{V_{0}\left(B_{1}\right)-V_{0}\left(B_{2}\right)}{2 A}+\frac{3}{4}$.

Since the equation

$$
\left(2^{m} \pi^{m / 2} \beta I+W_{\alpha}\right) f=0
$$

has only trivial solution in $\mathscr{B}$ provided $2^{m} \pi^{m / 2}|\beta|>\omega W_{\alpha}$, the last theorem implies the following corollary:

Theorem 4. If $D$ fulfils (3) then $H$ has a bounded inverse on $\mathscr{B}$.

As a by-product one obtains also a theorem on integral representation of solutions of the first problem of Fourier for the equation

(see theorem 3.11 below).

$$
\sum_{j=1}^{m} \partial_{j}^{2} u+\partial_{m+1} u=0
$$

\section{CHAPTER 1}

In this chapter we shall prove several results related to theorem 1 announced in the introduction.

1.1. Notation. $N$ is the set of all positive integers. If $M$ is a subset in some Euclidean space (whose dimension will always be clear from the context) then the symbols cl $M$, int $M$, fr $M$ and $\operatorname{diam} M$ will denote the closure, interior, boundary and diameter of $M$, respectively. Further let $H_{k} M$ stand for the outer Hausdorff $k$-dimensional measure of $M$ defined by

$$
H_{k} M=2^{-k} \alpha(k) \lim _{\varepsilon \rightarrow 0+} \inf \sum_{n}\left(\operatorname{diam} M_{n}\right)^{k},
$$

where

$$
\alpha(k)=\pi^{k / 2} / \Gamma\left(1+\frac{1}{2} k\right)
$$

is the volume of the unit $k$-ball and the infimum in (4) is taken over all sequences $\left\{M_{n}\right\}_{n \in N}$ of sets $M_{n}$ with $\bigcup_{n} M_{n}=M$ such that diam $M_{n} \leqq \varepsilon$ for all $n \in N$. If $M \subset R^{k}$ (= the Euclidean $k$-space), then $H_{k} M$ coincides with the outer Lebesgue measure of $M$. The support of a function $f$ (with domain in some Euclidean space) will be denoted by spt $f$.

The following simple remarks will be useful below. 
1.2. Remarks. Fix an infinitely differentiable function $\omega$ in $R^{1}$ with spt $\omega \subset$ $\subset(-1,1)$ such that

$$
\int_{R^{1}} \omega \mathrm{d} H_{1}=1, \omega(-r)=\omega(r), \quad r \in R^{1} .
$$

For each locally integrable function $g$ in $R^{1}$ and each $n \in N$ define

$$
A_{n} g(t)=n \int_{R^{1}} g(t-r) \omega(n r) \mathrm{d} r .
$$

Then $A_{n} g$ is infinitely differentiable and for each integrable function $\psi$ with compact support in $R^{1}$

$$
\int_{R^{1}} \psi A_{n} g \mathrm{~d} H_{1}=\int_{R^{1}} g A_{n} \psi \mathrm{d} H_{1} .
$$

Let now $Z$ be a non-void set. For each function $f$ on $R^{1} \times Z$ and each $z \in Z$ define $f_{z}$ on $R^{1}$ by

$$
f_{z}(t)=f(t, z), \quad t \in R^{1} .
$$

If $f_{z}$ happens to be locally integrable for each $z \in Z$ we define $A_{n} f$ on $R^{1} \times Z$ by

$$
\left(A_{n} f\right)_{z}=A_{n} f_{z}, \quad z \in Z, \quad n \in N .
$$

If the derivative $\left(f_{z}\right)^{\prime}$ exists in $R^{1}$ for each $z \in Z$ then $\partial f$ will denote the corresponding partial derivative in $R^{1} \times Z$ given by

$$
(\partial f)_{z}=\left(f_{z}\right)^{\prime}, \quad z \in Z .
$$

It is easily seen that, for each $n \in N$,

$$
A_{n} \partial f=\partial A_{n} f
$$

provided $\left(f_{z}\right)^{\prime}$ is locally integrable in $R^{1}$ for each $z \in Z$.

Suppose now that $\boldsymbol{A}$ is a $\sigma$-algebra of subsets in $Z$ and denote by $\boldsymbol{B}$ the $\sigma$-algebra of all Borel sets in $R^{1}$. If $h$ is $\mathbf{B} \times \mathbf{A}$-measurable on $R^{1} \times Z$ and $h_{z}$ is integrable for each $z \in Z$ then the integral

$$
\int_{R^{1}} h(t, z) \mathrm{d} H_{1}(t)
$$

represents an $\boldsymbol{A}$-measurable function of the variable $z \in Z$. Applying this to

$$
h\left(t, z_{1}, z_{2}\right)=n f\left(z_{1}-t, z_{2}\right) \omega(n t)\left(z_{1}, t \in R^{1}, z_{2} \in Z\right)
$$

with $Z$ replaced by $R^{1} \times Z, z$ replaced by $\left[z_{1}, z_{2}\right]$ and $\boldsymbol{A}$ replaced by $\mathbf{B} \times \boldsymbol{A}$, one easily obtains that $A_{n} f$ is $\mathbf{B} \times \mathbf{A}$-measurable provided $f$ is a $\mathbf{B} \times \mathbf{A}$-measurable func- 
tion on $R^{1} \times Z$ such that $f_{z}$ is locally integrable for each $z \in Z$. Consequently, for such an $f$ also $\partial A_{n} f$ is $\mathbf{B} \times \mathbf{A}$-measurable.

1.3. Lemma. Let us keep the notation of 1.2 and let $\lambda \geqq 0$ be a measure on $\boldsymbol{A}$. For each $k \in N$ let $\Psi_{k}$ be a class of $\mathbf{B} \times$ A-measurable functions on $R^{1} \times Z$ enjoying the following properties:

$\left(\mathrm{P}_{1}\right) \Psi_{k} \subset \Psi_{k+1}, k \in N$.

$\left(\mathrm{P}_{2}\right) \psi \in \Psi_{k} \Rightarrow-\psi \in \Psi_{k}$.

$\left(\mathrm{P}_{3}\right)$ For each $\psi \in \Psi=\bigcup_{k \in N} \Psi_{k}$ both $\partial \psi$ and $\psi$ are integrable $\left(H_{1} \times \lambda\right)$ and, for each $z \in Z, \psi_{z}$ is a continuously differentiable function with compact support in $R^{1}$.

$\left(\mathrm{P}_{4}\right)$ Given $k \in N$, there is a $n_{k} \in N$ such that

$$
\left(\psi \in \Psi_{k}, n \geqq n_{k}\right) \Rightarrow A_{n} \psi \in \Psi
$$

$\left(\mathrm{P}_{5}\right)$ For each $k$ there is a $G_{k} \in \mathbf{B} \times \mathbf{A}$ such that, for each bounded $\mathbf{B} \times \mathbf{A}$ measurable $h$ on $R^{1} \times Z$,

$$
\sup \left\{\int_{R^{1} \times Z} h \psi \mathrm{d}\left(H_{1} \times \lambda\right) ; \psi \in \Psi_{k}\right\}=\int_{G_{k}}|h| \mathrm{d}\left(H_{1} \times \lambda\right) .
$$

( $\left.\mathrm{P}_{6}\right)$ If $g$ is a bounded B-measurable function on $R^{1}$ then, for each $z \in Z$ and $k \in N$,

where

$$
\sup \left\{\int_{R^{1}} g \psi_{z} \mathrm{~d} H_{1} ; \psi \in \Psi_{k}\right\}=\int_{G_{k z}}|g| \mathrm{d} H_{1}
$$

$$
G_{k z}=R^{1} \cap\left\{t ;[t, z] \in G_{k}\right\}
$$

Suppose now that $f$ is a bounded B $\times$ A-measurable function on $R^{1} \times Z$ and let

$$
F(z)=\sup \left\{\int_{R^{1}} f_{z}(\partial \dot{\psi})_{z} \mathrm{~d} H_{1} ; \psi \in \Psi\right\}, \quad z \in Z .
$$

Then $F$ is a non-negative A-measurable function of the variable $z \in Z$ and

$$
\int_{z} F \mathrm{~d} \lambda=\sup \left\{\int_{R^{1} \times Z} f \partial \psi \mathrm{d}\left(H_{1} \times \lambda\right) ; \psi \in \Psi\right\} .
$$

Proof. Fix $z \in Z$. In view of $\left(\mathrm{P}_{4}\right)$, we have for $k \in N$ and $n \geqq n_{k}$

$$
\sup \left\{\int_{R^{1}} f_{z}\left(\partial A_{n} \psi\right)_{z} \mathrm{~d} H_{1} ; \psi \in \Psi_{k}\right\}=F_{k n}(z) \leqq F(z),
$$


whence it follows

$$
\underline{F}_{k}(z)=\liminf _{n \rightarrow \infty} F_{k n}(z) \leqq \limsup _{n \rightarrow \infty} F_{k n}(z)=\bar{F}_{k}(z) \leqq F(z) .
$$

In view of $\left(\mathbf{P}_{1}\right)$

$$
k \in N \Rightarrow \underline{F}_{k}(z) \leqq \underline{F}_{k+1}(z), \quad \bar{F}_{k}(z) \leqq \bar{F}_{k+1}(z)
$$

and we conclude that

$$
\lim _{k \rightarrow \infty} \bar{F}_{k}(z) \leqq F(z) .
$$

On the other hand, if $c<F(z)$, then there is a $\psi \in \Psi$ with

$$
\int_{R^{1}} f_{z}(\partial \psi)_{z} \mathrm{~d} H_{1}>c
$$

Noting that all the functions in $\left\{A_{n}(\partial \psi)_{z}\right\}_{n \in N}$ have support in a fixed compact subset of $R^{1}$ and converge uniformly to $(\partial \psi)_{z}$ as $n \rightarrow \infty\left(\right.$ cf. $\left.\left(\mathrm{P}_{3}\right)\right)$ we get for $k \in N$ with $\Psi_{k} \ni \psi$

$$
\underline{F}_{k}(z) \geqq \int_{R^{1}} f_{z}(\partial \psi)_{z} \mathrm{~d} H_{1}>c
$$

We have thus proved

$$
F(z)=\lim _{k \rightarrow \infty} \underline{F}_{k}(z)=\lim _{k \rightarrow \infty} \bar{F}_{k}(z) .
$$

Employing the remarks in 1.2 we see that, for $\psi \in \Psi_{k}$ and $n \geqq n_{k}$,

$$
\int_{R^{1}} f_{z}\left(\partial A_{n} \psi\right)_{z} \mathrm{~d} H_{1}=\int_{R^{1}} f_{z}\left(A_{n} \partial \psi\right)_{z} \mathrm{~d} H_{1}=-\int_{R^{1}}\left(\partial A_{n} f\right)_{z} \psi_{z} \mathrm{~d} H_{1},
$$

whence it follows by $\left(\mathrm{P}_{6}\right),\left(\mathrm{P}_{2}\right)$ for $n \geqq n_{k}$

$$
F_{k n}(z)=\int_{G_{k z}}\left|\left(\partial A_{n} f\right)_{z}\right| \mathrm{d} H_{1},
$$

which is an A-measurable function of $z \in Z$. Consequently, also $\underline{F}_{k}$ and $F=\lim _{k \rightarrow \infty} \underline{F}_{k}$ are $\boldsymbol{A}$-measurable non-negative functions. It remains to verify

$$
\int_{\mathrm{Z}} F \mathrm{~d} \lambda \leqq \sup \left\{\int_{R^{1} \times \mathrm{Z}} f \partial \psi \mathrm{d}\left(H_{1} \times \lambda\right) ; \psi \in \Psi\right\}=K,
$$

because the opposite inequality follows at once from the definition of $F$. We have by $(5),(6)$

$$
\int_{Z} F \mathrm{~d} \lambda=\lim _{k \rightarrow \infty} \int_{Z} \underline{F}_{k} \mathrm{~d} \lambda \leqq \lim _{k \rightarrow \infty} \liminf _{n \rightarrow \infty} \int_{Z} F_{k n} \mathrm{~d} \lambda
$$


Employing (7) and $\left(\mathrm{P}_{5}\right)$ we get

$$
\int_{Z} F_{k n} \mathrm{~d} \lambda=\sup \left\{\int_{R^{1} \times Z}\left(\partial A_{n} f\right) \psi \mathrm{d}\left(H_{1} \times \lambda\right) ; \psi \in \Psi_{k}\right\} .
$$

Now it is sufficient to observe that, for $\psi \in \Psi_{k}$ and $n \geqq n_{k}$

$$
\int_{R^{1} \times Z}\left(\partial A_{n} f\right) \psi \mathrm{d}\left(H_{1} \times \lambda\right)=-\int_{R^{1} \times Z} f \partial A_{n} \psi \mathrm{d}\left(H_{1} \times \lambda\right) \leqq K
$$

by $1.2,\left(\mathrm{P}_{4}\right)$ and $\left(\mathrm{P}_{2}\right)$.

1.4. Remark. The above lemma, which is in fact an abstract version of lemma 1.10 in $[10]$, is closely connected with investigations of functions whose partial derivatives are measures; see [1], [6], [9], [12], [15], [16], [18].

1.5. Notation. As in the introduction, $\Omega_{\varrho}(y)$ will denote the ball of center $y$ and radius $\varrho$ in $R^{m}$, and $\Gamma=\operatorname{fr} \Omega_{1}(0)$. It is convenient to adopt the following terminology introduced in [10], 1.5: If $S$ is an open segment or half-line in $R^{m}$ then $y \in S$ is termed a hit of $S$ on $D$ provided

$$
H_{1}\left(\Omega_{\varrho}(y) \cap S \cap D\right)>0 \text { and } H_{1}\left(S \cap \Omega_{\varrho}(y) \backslash D\right)>0
$$

for each $\varrho>0$. The number of all hits of

$$
S_{r}(x)=\{x+\varrho \theta ; 0<\varrho<r\}\left(x \in R^{m}, \theta \in \Gamma\right)
$$

on $D$ will be denoted by $n_{r}(\theta, x)\left(0 \leqq n_{r}(\theta, x) \leqq+\infty\right)$. According to 1.6 in [10], $n_{r}(\theta, x)$ is a Baire function of the variable $\theta \in \Gamma$ and we let

$$
v(r ; x)=\int_{\Gamma} n_{r}(\theta, x) \mathrm{d} H_{m-1}(\theta) .
$$

For the sake of brevity we shall sometimes write $v_{r}(x)$ instead of $v(r ; x)$.

$\mathscr{D}$ will stand for the class of all infinitely differentiable functions with compact support in $R^{m+1}$. For $T \in(-\infty,+\infty)$ let

$$
R_{T}=R^{m} \times(-\infty, T)
$$

and denote by $\mathscr{D}_{T}$ the class of all $\varphi \in \mathscr{D}$ with spt $\varphi \subset R_{T}$. The derivative with respect to the $j$-th variable will be denoted by $\partial_{j}$. The points $z=\left[z_{1}, \ldots, z_{m+1}\right] \in R^{m+1}$ will often be written in the form $\left[\hat{z}, z_{m+1}\right]$ with $\hat{z}=\left[z_{1}, \ldots, z_{m}\right] \in R^{m}$. We shall write

$$
\hat{\nabla}=\left[\partial_{1}, \ldots, \partial_{m}\right] \text {. }
$$


The Euclidean norm is denoted by $|\ldots|$. As in the introduction, we denote by $G$ the well-known kernel connected with the heat equation, defining $G=0$ on $\mathrm{cl} R_{0}$ and letting

$$
G(x, t)=t^{-m / 2} \exp \left(-|x|^{2} / 4 t\right) \text { for }[x, t] \in R^{m} \times(0,+\infty) .
$$

Simple calculation shows that for

$$
R_{\alpha \beta}=R_{\beta} \backslash R_{\alpha}, \quad-\infty<\alpha<\beta<+\infty,
$$

the following estimates hold:

$$
\begin{gathered}
\int_{R_{\alpha \beta}}\left|\partial_{j} G\right| \mathrm{d} H_{m+1} \leqq 2^{m+1}\left[\pi^{m-1}(\beta-\alpha)\right]^{1 / 2}, \quad 1 \leqq j \leqq m, \\
\int_{R_{\alpha \beta}} G \mathrm{~d} H_{m+1} \leqq 2^{m} \pi^{m / 2}(\beta-\alpha) .
\end{gathered}
$$

By the term measure we shall usually mean a finite signed Borel measure defined on the $\sigma$-algebra of all Borel subsets of a fixed Borel set in some Euclidean space. If $\mu$ is a measure and $M$ is a Borel set in the domain of $\mu$, then $|\mu|(M)$ denotes the variation of $\mu$ on $M$; spt $\mu$ will denote the support of $\mu$.

Let $D \subset R^{m}$ be an open set with a compact boundary $B \neq \emptyset$. Fix now $T_{1}, T_{2}$, $-\infty<T_{1} \leqq T_{2} \leqq+\infty$, and put

$$
E=D \times\left(T_{1}, T_{2}\right), \quad C=B \times\left\langle T_{1}, T_{2}\right) .
$$

Denote by $\mathscr{B}^{\prime}=\mathscr{B}^{\prime}\left(T_{1}, T_{2}\right)$ the Banach space of all measures $\mu$ in $R^{m+1}$ with

$$
|\mu|\left(R^{m+1} \backslash C\right)=0 ;
$$

the norm in $\mathscr{B}^{\prime}$ is given by

$$
\|\mu\|=|\mu|(C) .
$$

With each $\mu \in \mathscr{B}^{\prime}$ associate the potential

$$
U \mu(z)=\int G(z-\zeta) \mathrm{d} \mu(\zeta), \quad z \in R^{m+1} \backslash \operatorname{cl} C
$$

Then $U \mu$ is an infinitely differentiable function on $R^{m+1} \backslash \mathrm{cl} C$ satisfying there the heat equation

$$
\sum_{j=1}^{m} \partial_{j}^{2} U \mu=\partial_{m+1} U \mu
$$

Employing (10), (11) one obtains at once for

$$
E_{\alpha \beta}=E \cap\left(R_{\beta} \backslash R_{\alpha}\right), \quad-\infty<\alpha<\beta<+\infty,
$$


that

$$
\begin{gathered}
\int_{E_{\alpha \beta}}\left|\partial_{j} U \mu\right| \mathrm{d} H_{m+1} \leqq 2^{m+1}\left[\pi^{m-1}(\beta-\alpha)\right]^{1 / 2}\|\mu\|, \quad 1 \leqq j \leqq m \\
\int_{E_{\alpha \beta}}|U \mu| \mathrm{d} H_{m+1} \leqq 2^{m} \pi^{m / 2}(\beta-\alpha)\|\mu\| .
\end{gathered}
$$

Accordingly, we are justified to introduce the distribution $H \mu$ in $R_{T_{2}}$ defining for $\varphi \in \mathscr{D}_{T_{2}}$

$$
\langle\varphi, H \mu\rangle=\int_{E}\left(\hat{\nabla} U \mu \cdot \hat{\nabla} \varphi-U \mu . \partial_{m+1} \varphi\right) \mathrm{d} H_{m+1} .
$$

As it is usual in distribution theory [23], we shall say that $H \mu$ is a measure provided there is a measure $v_{\mu}$ in $R^{m+1}$ such that

$$
\langle\varphi, H \mu\rangle=\int \varphi \mathrm{d} v_{\mu}, \quad \varphi \in \mathscr{D}_{T_{2}} .
$$

It is easily seen that (15) together with

$$
\left|v_{\mu}\right|\left(R^{m+1} \backslash R_{T_{2}}\right)=0
$$

determine $v_{\mu}$ uniquely and that each $v_{\mu}$ enjoying (15), (16) satisfies

$$
\text { spt } v_{\mu} \subset \operatorname{cl} C \text {. }
$$

Indeed, if $\varphi \in \mathscr{D}_{T_{2}}$ and spt $\varphi \cap \mathrm{cl} C=\emptyset$, then there is a bounded open set $\tilde{D} \subset R^{m}$ with cl $\widetilde{D} \subset D$ such that the boundary of $\widetilde{D}$ is a smooth hypersurface $\widetilde{B}$ and

$$
E \cap \operatorname{spt} \varphi \subset \tilde{D} \times\left(T_{1}, T_{2}\right)=\tilde{E} .
$$

Taking into account (12) (note also that $U \mu$ vanishes on $D \times\left\{T_{1}\right\}$ and $\varphi$ vanishes on $\left.D \times\left\{T_{2}\right\}\right)$ and writing $\tilde{n}$ for the exterior normal of $\widetilde{D}$ we obtain by the GaussGreen theorem

$$
\begin{aligned}
& \langle\varphi, H \mu\rangle=\int_{\tilde{E}}\left(\hat{\nabla} U \mu \cdot \hat{\nabla} \varphi-U \mu \cdot \partial_{m+1} \varphi\right) \mathrm{d} H_{m+1}= \\
& =\int_{T_{1}}^{T_{2}} \mathrm{~d} t \int_{\tilde{B}} \varphi(x, t) \tilde{n}(x) \cdot \hat{\nabla} U \mu(x, t) \mathrm{d} H_{m-1}(x)=0 .
\end{aligned}
$$

We conclude from (16), (17) that $v_{\mu} \in \mathscr{B}^{\prime}$.

1.6. Lemma. Given $\zeta=[\xi, \tau] \in R^{m+1}$ and $\varphi \in \mathscr{D}$ let

$$
\tilde{W} \varphi(\zeta)=\int_{E}\left[\hat{\nabla} G(z-\zeta) \cdot \hat{\nabla} \varphi(z)-G(z-\zeta) \partial_{m+1} \varphi(z)\right] d z
$$


and define $S \varphi$ on $(0,+\infty) \times(0,+\infty) \times \Gamma$ by

$$
S \varphi(\varrho, \eta, \theta)=\varphi\left(\xi+\varrho \theta, \tau+\varrho^{2} / 4 \eta\right), \quad \varrho, \eta \in(0,+\infty), \quad \theta \in \Gamma .
$$

If $\tau \in\left\langle T_{1}, T_{2}\right)$ then

$$
\widetilde{W} \varphi(\zeta)=-2^{m-1} \int_{\Gamma} \mathrm{d} H_{m-1}(\theta) \int_{0}^{\infty} \mathrm{e}^{-\eta} \eta^{m / 2-1} \mathrm{~d} \eta \int_{D_{*}} \partial_{1} S \varphi(\varrho, \eta, \theta) \mathrm{d} \varrho,
$$

where

$$
D_{*}=\left\{\varrho ; 0<\varrho<2\left[\eta\left(T_{2}-\tau\right)\right]^{1 / 2}, \xi+\varrho \theta \in D\right\} .
$$

Proof. Simple calculation yields

$$
\widetilde{W} \varphi(\zeta)=-\frac{1}{2} \int_{\tau}^{T_{2}}(t-\tau)^{-m / 2-1} \mathscr{J}(t) \mathrm{d} t,
$$

where

$$
\mathscr{J}(t)=\int_{D} \mathrm{e}^{-|x-\xi|^{2 / 4(t-\tau)}}\left[(x-\xi) \cdot \hat{\nabla} \varphi(x, t)+2(t-\tau) \partial_{m+1} \varphi(x, t)\right] \mathrm{d} x .
$$

Let us now introduce the variables $r \in(0,+\infty)$ and $\theta \in \Gamma$ by

$$
x=\xi+r \theta .
$$

Then $\mathrm{d} x=r^{m-1} \mathrm{~d} r \mathrm{~d} H_{m-1}(\theta)$ and $\mathscr{J}(t)$ transforms into

$$
\int_{\Gamma} \mathscr{K}(t, \theta) \mathrm{d} H_{m-1}(\theta)=\mathscr{J}(t),
$$

where $\mathscr{K}(t, \theta)$ denotes the integral extended over

given by

$$
D_{\theta}=\{r ; r>0, \xi+r \theta \in D\}
$$

$$
\mathscr{K}(t, \theta)=\int_{D_{\theta}} \mathrm{e}^{-r^{2} / 4(t-\tau)}\left[r \frac{\partial \varphi(\xi+r \theta, t)}{\partial r}+2(t-\tau) \frac{\partial \varphi(\xi+r \theta, t)}{\partial t}\right] r^{m-1} \mathrm{~d} r .
$$

Consequently,

$$
\tilde{W} \varphi(\zeta)=-\frac{1}{2} \int_{\Gamma} \mathscr{L}(\theta) \mathrm{d} H_{m-1}(\theta)
$$

where

$$
\mathscr{L}(\theta)=\int_{\tau}^{T_{2}}(t-\tau)^{-m / 2-1} \mathscr{K}(t, \theta) \mathrm{d} t=\iint \ldots \mathrm{d} r \mathrm{~d} t
$$


may be considered as a double integral extended over $[r, t] \in D_{\theta} \times\left(\tau, T_{2}\right)$. Employing the change of variables

$$
r=\varrho, \quad t=\tau+\frac{\varrho^{2}}{4 \eta}
$$

we get after simple calculation

$$
\mathscr{L}(\theta)=2^{m} \int_{0}^{\infty} \mathrm{e}^{-\eta} \eta^{m / 2-1} \mathrm{~d} \eta \int_{D_{*}} \partial_{1} S \varphi(\varrho, \eta, \theta) \mathrm{d} \varrho,
$$

which completes the proof.

1.7. Remark. Let $\delta_{\zeta}$ denote the unit point mass (= Dirac measure) concentrated at $\zeta$. Noting that

$$
G(z-\zeta)=U \delta_{\zeta}(z)
$$

we observe that

$$
\tilde{W} \varphi(\zeta)=\left\langle\varphi, H \delta_{\zeta}\right\rangle
$$

provided $\varphi \in \mathscr{D}_{T_{2}}$ and $\zeta \in C$.

1.8. Proposition. Let $\zeta=[\xi, \tau] \in R^{m+1}, T_{1} \leqq \tau<T_{2}$, fix $R>0, \varepsilon>0$ and put

$$
\begin{gathered}
\mathscr{D}^{1}=\mathscr{D}_{T_{2}} \cap\left\{\varphi ;|\varphi| \leqq 1, \operatorname{spt} \varphi \subset\left[\Omega_{R}(\xi) \backslash\{\xi\}\right] \times(\tau, \tau+\varepsilon)\right\}, \\
r(\eta)=\min \left\{R, 2\left[\eta \min \left(\varepsilon, T_{2}-\tau\right)\right]^{1 / 2}\right\}, \quad \eta>0 .
\end{gathered}
$$

Then

$$
2^{m-1} \int_{0}^{\infty} \mathrm{e}^{-\eta} \eta^{m / 2-1} v(r(\eta) ; \xi) \mathrm{d} \eta=\sup \left\{\tilde{W} \varphi(\zeta) ; \varphi \in \mathscr{D}^{1}\right\}
$$

Proof. We shall apply lemma 1.3 . Define the measure $\mu$ by

$$
\mathrm{d} \mu(\eta)=2^{m-1} \mathrm{e}^{-\eta} \eta^{m / 2-1} \mathrm{~d} H_{1}(\eta)
$$

and consider the product measure $\lambda=\mu \times H_{m-1}$ on the $\sigma$-algebra of all Borel subsets of $Z=(0, \infty) \times \Gamma$. It is easily seen that the mapping

$$
\Phi:[\varrho, \eta, \theta] \rightarrow\left[\xi+\varrho \theta, \tau+\frac{\varrho^{2}}{4 \eta}\right]
$$

maps $(0, \infty) \times(0, \infty) \times \Gamma=(0, \infty) \times Z$ homeomorphically onto

$$
\left[R^{m} \backslash\{\xi\}\right] \times(\tau, \infty) .
$$


Let

$$
c=\min \left(\varepsilon, T_{2}-\tau\right), \tilde{E}=\left[\Omega_{R}(\xi) \backslash\{\xi\}\right] \times(\tau, \tau+c),
$$

define $r(\eta)$ by $(22)$ and put

$$
G=\Phi^{-1}(\widetilde{E})=\{[\varrho, \eta] ; \eta>0,0<\varrho<r(\eta)\} \times \Gamma .
$$

Fix a decreasing sequence of positive numbers $\left\{\varepsilon_{k}\right\}_{k=1}^{\infty}$ such that

and define

$$
2 \varepsilon_{1}<R, \lim _{k \rightarrow \infty} \varepsilon_{k}=0
$$

$$
G_{k}=\left\{[\varrho, \eta] ; \eta>\varepsilon_{k}^{2} c^{-1}, \varepsilon_{k}<\varrho<r(\eta)-\varepsilon_{k}\right\} \times \Gamma .
$$

Denote by $\Psi_{k}$ the class of all functions $\psi$ with domain $X=R^{1} \times Z$ for which there is a $\varphi \in \mathscr{D}^{1}$ (depending on $\left.\psi\right)$ such that

$$
\text { spt } S \varphi \subset G_{k}, \quad \psi=S \varphi \text { in } G
$$

and

$$
\psi(X \backslash G)=\{0\}
$$

Then the class of all (point-wise) limits of sequences of elements of $\Psi$ coincides with the class of all the functions $g$ of the first class of Baire on $X$ such that,

$$
|g| \leqq 1, \quad X \backslash G_{k} \subset g^{-1}(0) .
$$

Hence we conclude that the conditions $\left(\mathrm{P}_{6}\right),\left(\mathrm{P}_{5}\right)$ in 1.3 are satisfied. Fix now $n_{k}>\varepsilon_{k}^{-1}$. If $\psi \in \Psi_{k}, \psi=S \varphi$ in $G$ and $A_{l} \psi$ with $n \geqq n_{k}$ is defined by 1.2 , then $A_{n} \psi$ has a compact support contained in $G$. Simple calculation shows that the value attained by $\left(A_{n} \psi\right)$ 。 - $\Phi^{-1}\left(=\right.$ the composite of $\Phi^{-1}$ and $\left.A_{n} \psi\right)$ at $[x, t] \in\left[R^{m} \backslash\{\xi\}\right] \times(\tau, \infty)$ is given by the integral

$$
\int_{R^{1}} n \varphi\left(h(u, x)(x-\xi)+\xi, \quad h^{2}(u, x)(t-\tau)+\tau\right) \omega(n u) \mathrm{d} u,
$$

where $\omega$ has the meaning described in 1.2 and

$$
h(u, x)=\frac{|x-\xi|-u}{|x-\xi|} .
$$

Defining $\tilde{\varphi}(x, t)$ by $(23)$ for $[x, t] \in \widetilde{E}$ and letting

$$
\tilde{\varphi}\left(R^{m+1} \backslash \widetilde{E}\right)=\{0\}
$$

we see that $\tilde{\varphi} \in \mathscr{D}^{1}$,

$$
A_{n} \psi=\tilde{\varphi} \circ \Phi=S \tilde{\varphi} \text { in } \quad G
$$


Consequently, $A_{n} \psi \in \Psi=\bigcup_{k=1}^{\infty} \Psi_{k}$ and $\left(\mathrm{P}_{4}\right)$ is verified. The conditions $\left(\mathrm{P}_{1}\right),\left(\mathrm{P}_{2}\right),\left(\mathrm{P}_{3}\right)$ being obviously fulfilled we are justified to apply 1.3 to the characteristic function $f$ of $G$. Employing 1.6 we get

$$
\begin{gathered}
\sup \left\{\tilde{W} \varphi(\zeta) ; \varphi \in \mathscr{D}^{1}\right\}= \\
\sup \left\{\int_{X} f \partial_{1} \psi \mathrm{d}\left(H_{1} \times \lambda\right) ; \psi \in \Psi\right\}=\int_{z} F \mathrm{~d} \lambda
\end{gathered}
$$

where, for fixed $z=[\eta, \theta] \in Z$,

$$
F(z)=\sup \left\{\int_{R^{1}} f_{z}\left(\partial_{1} \psi\right)_{z} \mathrm{~d} H_{1} ; \psi \in \Psi\right\} .
$$

Note that $\left\{\psi_{z} ; \psi \in \Psi\right\}$ coincides with the class of all infinitely differentiable functions $\gamma$ in $R^{1}$ with

$$
|\gamma| \leqq 1, \quad \text { spt } \gamma \subset(0, r(\eta))
$$

Taking into account that $f_{z}$ is the characteristic function of $D_{*} \cap(0, r(\eta))$, where $D_{*}$ is defined by (19), we conclude from 1.9 in [10] that $F(z)$ equals the number of hits of $(0, r(\eta))$ on $D_{*}$. In other words, $F(z)$ is the number of hits of

$$
\{\xi+\varrho \theta ; 0<\varrho<r(\eta)\}
$$

on $D$ and, consequently,

$$
\int_{\Gamma} F(\eta, \theta) \mathrm{d} H_{m-1}(\theta)=v(r(\eta) ; \xi),
$$

which completes the proof.

1.9. Lemma. Fix $\zeta=[\xi ; \tau] \in C$. If $H \delta_{\zeta}$ is a measure then

$$
v_{\infty}^{\prime}(\xi)<\infty \text {. }
$$

Conversely, if (24) holds, then $H \delta_{\zeta}$ may be identified with an element of $\mathscr{B}^{\prime}$ and its norm admits the estimates

$$
\begin{aligned}
&\left\|H \delta_{\zeta}\right\| \leqq 2^{m-1}\left[v_{\infty}(\xi) \Gamma\left(\frac{1}{2} m\right)+2 \pi^{m / 2}\right], \\
&\left\|H \delta_{\zeta}\right\| \geqq 2^{m-1} v_{\infty}(\xi) \int_{b}^{\infty} \mathrm{e}^{-\eta} \eta^{m / 2-1} \mathrm{~d} \eta,
\end{aligned}
$$

where

$$
b=\frac{(\operatorname{diam} B)^{2}}{4\left(T_{2}-\tau\right)}
$$


Proof. Let $R=+\infty=\varepsilon$ and define $\mathscr{D}^{1}$ by (21) for this particular choice of $R$ and $\varepsilon$. Suppose first that $H \delta_{\zeta}$ is a measure. Then $H \delta_{\zeta} \in \mathscr{B}^{\prime}$ (see 1.5) and

$$
\begin{gathered}
\left\|H \delta_{\zeta}\right\| \geqq \sup \left\{\left\langle\varphi, H \delta_{\zeta}\right\rangle ; \varphi \in \mathscr{D}^{1}\right\}= \\
=2^{m-1} \int_{0}^{\infty} \mathrm{e}^{-\eta} \eta^{m / 2-1} v\left(2\left[\eta\left(T_{2}-\tau\right)\right]^{1 / 2} ; \xi\right) \mathrm{d} \eta \geqq 2^{m-1} v_{\infty}(\xi) \int_{b}^{\infty} \mathrm{e}^{-\eta} \eta^{m / 2-1} \mathrm{~d} \eta,
\end{gathered}
$$

because $v_{\varrho}(\xi)=v_{\infty}(\xi)$ for $\varrho>\operatorname{diam} B$.

Assume now (24) and consider $\varphi \in \mathscr{D}_{T_{2}},|\varphi| \leqq 1$. Fix $\theta \in \Gamma, \eta>0$ and define $D_{*}$, $S \varphi$ as in 1.6. We shall show that

$$
\left|\int_{D_{*}} \partial_{1} S \varphi(\varrho, \eta, \theta) \mathrm{d} \varrho\right| \leqq 1+n_{\infty}(\theta, \xi) .
$$

It is sufficient to consider the case when $n_{\infty}(\theta, \xi)<+\infty$. Let us agree to write simply $S(\varrho)=S \varphi(\varrho, \eta, \theta)$, so that $S^{\prime}(\varrho)=\partial_{1} S \varphi(\varrho, \eta, \theta)$. Put $r=2\left[\left(T_{2}-\tau\right) \eta\right]^{1 / 2}$ and let $\varrho_{1}<\ldots<\varrho_{n}$ be all the hits of $(0, r)$ on $D_{*}$. Further put $\varrho_{n+1}=r, \varrho_{0}=0$. Since $D_{*}$ is open and $\left(\varrho_{i-1}, \varrho_{i}\right)$ contains no hits on $D_{*}$,

$$
D_{i}=D_{*} \cap\left(\varrho_{i-1}, \varrho_{i}\right) \text { is either void and } \int_{D_{i}} S^{\prime}(\varrho) \mathrm{d} \varrho=0,
$$

or else $H_{1}\left(D_{i}\right)=\varrho_{i}-\varrho_{i-1}$, in which case

$$
\int_{D_{i}} S^{\prime}(\varrho) \mathrm{d} \varrho=S\left(\varrho_{i}\right)-S\left(\varrho_{i-1}\right) .
$$

Noting that $S\left(\varrho_{i}\right) \leqq 1$ for $0 \leqq i \leqq n$ and $S\left(\varrho_{n+1}\right)=0$, we conclude that

$$
\left|\int_{D_{*}} S^{\prime}(\varrho) \mathrm{d} \varrho\right| \leqq n+1
$$

The inequality (26) together with $1.6,1.7$ yields

$$
\sup \left\{\left\langle\varphi, H \delta_{\zeta}\right\rangle ; \varphi \in \mathscr{D}_{T_{2}},|\varphi| \leqq 1\right\} \leqq 2^{m-1}\left[2 \pi^{m / 2}+\Gamma\left(\frac{1}{2} m\right) v_{\infty}(\xi)\right]
$$

and the proof is complete.

1.10. Remark. If $\mu \in \mathscr{B}^{\prime}$ and $\varphi \in \mathscr{D}_{T_{2}}$, then

$$
\langle\varphi, H \mu\rangle=\int_{C}\left\langle\varphi, H \delta_{\zeta}\right\rangle \mathrm{d} \mu(\zeta)
$$


. Proof. Taking into account (10), (11) and applying Fubini's theorem to

$$
\iint_{E \times C}\left[\hat{\nabla} G(z-\zeta) \cdot \hat{\nabla} \varphi(z)-G(z-\zeta) \partial_{m+1} \varphi(z)\right] \mathrm{d} H_{m+1}(z) \mathrm{d} \mu(\zeta)
$$

one obtains (27) (see also 1.7, 1.6).

A reasoning similar to that used in the proof of theorem 1.13 in [10] permits now to establish the following

1.11. Theorem. $H \mu$ is a measure for each $\mu \in \mathscr{B}^{\prime}$ if and only if

$$
V=\sup \left\{v_{\infty}(\xi) ; \xi \in B\right\}<\infty .
$$

If (28) holds then, for each $\mu \in \mathscr{B}^{\prime}, H \mu$ may be identified with a uniquely determined element of $\mathscr{B}^{\prime}$, the operator $H: \mu \rightarrow H \mu$ is bounded on $\mathscr{B}^{\prime}$ and

$$
\|H\| \leqq 2^{m-1}\left[\Gamma\left(\frac{1}{2} m\right) V+2 \pi^{m / 2}\right] .
$$

'Proof. For each $\varphi \in \mathscr{D}_{T_{2}}$ define the functional $L_{\varphi}$ on $\mathscr{B}^{\prime}$ by

$$
\left\langle\mu, L_{\varphi}\right\rangle=\langle\varphi, H \mu\rangle, \quad \mu \in \mathscr{B}^{\prime} .
$$

Let spt $\varphi \subset R_{\beta} \backslash R_{\alpha},-\infty<\alpha<\beta<\infty$ and put

$$
c=2^{m} \max \left\{2\left[\pi^{m-1}(\beta-\alpha)\right]^{1 / 2}, \pi^{m / 2}(\beta-\alpha)\right\}, \quad k(\varphi)=c \sup _{i=1}^{m+1}\left|\partial_{i} \varphi\right| .
$$

We get from the definition of $\langle\varphi, H \mu\rangle$ and (13), (14)

$$
\left|\left\langle\mu, L_{\varphi}\right\rangle\right| \leqq k(\varphi)\|\mu\|
$$

so that each functional $L_{\varphi}$ is bounded on $\mathscr{B}^{\prime}$.

Let

$$
\mathscr{A}=\mathscr{D}_{T_{2}} \cap\{\varphi ;|\varphi| \leqq 1\} .
$$

If $H \mu$ is measure for each $\mu \in \mathscr{B}^{\prime}$, then the class of functionals $\left\{L_{\varphi}\right\}_{\varphi \in \mathscr{A}}$ is pointwise bounded on $\mathscr{B}^{\prime}$. Hence it follows by the uniform boundedness principle

$$
\sup \left\{\left\|L_{\varphi}\right\| ; \varphi \in \mathscr{A}\right\}=K<+\infty .
$$

In particular, for each $\zeta \in C$,

$$
\left\|H \delta_{\zeta}\right\|=\sup \left\{\left\langle\delta_{\zeta}, L_{\varphi}\right\rangle ; \varphi \in \mathscr{A}\right\} \leqq K .
$$

Consider now an arbitrary $\xi \in B$ and let $\xi=\left[\xi, T_{1}\right] \in C$. Defining

$$
b=\frac{(\operatorname{diam} B)^{2}}{4\left(T_{2}-T_{1}\right)}
$$


we obtain from $\left(25_{2}\right)$

$$
v_{\infty}(\xi) \leqq 2^{1-m}\left(\int_{b}^{\infty} \mathrm{e}^{-\eta} \eta^{m / 2-1} \mathrm{~d} \eta\right)^{-1} \cdot K
$$

and (28) is verified. Conversely, if (28) holds, then 1.10 and 1.9 imply

$$
\sup \{|\langle\varphi, H \mu\rangle| ; \varphi \in \mathscr{A}\} \leqq 2^{m-1}\left[V \Gamma\left(\frac{1}{2} m\right)+2 \pi^{m / 2}\right]\|\mu\|
$$

for each $\mu \in \mathscr{B}^{\prime}$. This completes the proof.

Remark. In connection with the above reasonings we wish to mention here the work of G. FICHERA [5] on applications of functional analysis to boundary value problems.

\section{CHAPTER 2}

We are now going to investigate more closely the function $\tilde{W} \varphi$ which has appeared in $1.6,1.7$ for the special case when $\varphi \in \mathscr{D}_{T_{2}}$.

2.1. Lemma. Fix $\xi \in R^{m}$ with $v_{\infty}(\xi)<+\infty$ and define $s(\varrho ; \xi, \theta)$ for $\varrho>0$ and $\theta \in \Gamma$ as follows (compare 2.4 in [10]):

$$
s(\varrho ; \xi, \theta)=\sigma(= \pm 1)
$$

if there is a $\delta>0$ such that

$$
\xi+(\varrho+\sigma u) \theta \in D, \quad \xi+(\varrho-\sigma u) \theta \in R^{m} \backslash D
$$

for almost every $u \in(0, \delta)$; otherwise we set $s(\varrho ; \xi, \theta)=0$. Further fix $\tau \in\left\langle T_{1}, T_{2}\right)$; put $\zeta=[\xi, \tau]$ and associate with each bounded Baire function $f$ on $C$ the function $\sum_{f}(\zeta ; \eta, \theta)$ defined for $\theta \in \Gamma$ and $\eta>0$ as follows: If $n_{\infty}(\theta, \xi)<+\infty$ then

$$
\sum_{f}(\zeta ; \eta, \theta)=\sum_{\varrho} f\left(\xi+\varrho \theta, \tau+\frac{\varrho^{2}}{4 \eta}\right) s(\varrho ; \xi, \theta),
$$

the sum on the right - hand side being extended over $\varrho$ satisfying

$$
0<\varrho<2\left[\eta\left(T_{2}-\tau\right)\right]^{1 / 2}, s(\varrho ; \xi ; \theta) \neq 0 ;
$$

if $n_{\infty}(\theta, \xi)=+\infty$, we set

$$
\sum_{f}(\zeta ; \eta, \theta)=0 .
$$

Then $\sum_{f}(\zeta ; \eta, \theta)$ is integrable $\mathrm{d} H_{m-1}(\theta)$ over $\Gamma$ for each $\eta>0$. Besides that,

$$
V_{f}(\zeta ; \eta)=\int_{\Gamma} \sum_{f}(\zeta ; \eta, \theta) \mathrm{d} \boldsymbol{H}_{m-1}(\theta)
$$


is a bounded Baire function of the variable $\eta>0$. We are thus justified to define

$$
W f(\zeta)=2^{m-1} \int_{0}^{\infty} \mathrm{e}^{-\eta} V_{f}(\zeta ; \eta) \eta^{m / 2-1} \mathrm{~d} \eta
$$

Remark. If $F$ is a function whose domain contains $C$, then $W F$ is taken to mean $W f$, where $f$ is the restriction of $F$ to $C$.

Proof of lemma 2.1. Denote by $K_{\xi}$ the set of those $\theta \in \Gamma$, for which there is an $\varepsilon=\varepsilon(\theta)>0$ such that

$$
H_{1}(\{\xi+\varrho \theta ; 0<\varrho<\varepsilon\} \backslash D)=0,
$$

and consider first

$$
\theta \in K_{\xi}, \quad n_{\infty}(\theta, \xi)<+\infty
$$

Fix $\eta>0$, put

$$
r(\eta)=2\left[\eta\left(T_{2}-\tau\right)\right]^{1 / 2}
$$

and define

$$
D_{*}=\{\varrho ; 0<\varrho<r(\eta), \xi+\varrho \theta \in D\} .
$$

If $\varrho_{1}<\ldots<\varrho_{n}$ are all the hits of $(0, r(\eta))$ on $D_{*}$, then

$$
\begin{gathered}
s\left(\varrho_{j+1} ; \xi, \theta\right)=-s\left(\varrho_{j} ; \xi, \theta\right) \text { for } 1 \leqq j<n, \\
s\left(\varrho_{1} ; \xi, \theta\right)=-1 .
\end{gathered}
$$

Letting $\varrho_{0}=0$ we conclude for

$$
S(\varrho)=S \varphi(\varrho, \eta, \theta)
$$

defined by (18) that

$$
\int_{D^{*}} S^{\prime}(\varrho) \mathrm{d} \varrho=\sum_{j=0}^{n}(-1)^{j-1} S\left(\varrho_{j}\right)=-\varphi(\zeta)-\sum_{\varphi}(\zeta ; \eta, \theta) .
$$

We have thus shown for $\theta$ satisfying (29) that

$$
\int_{D^{*}} \partial_{1} S_{\varphi}(\varrho, \eta, \theta) \mathrm{d} \varrho=-\varphi(\zeta)-\sum_{\varphi}(\zeta ; \eta, \theta) .
$$

A similar reasoning shows for $\theta$ satisfying

$$
\theta \in \Gamma \backslash K_{\xi}, \quad n_{\infty}(\theta, \xi)<+\infty
$$


that

$$
\int_{D^{*}} \partial_{1} S \varphi(\varrho, \eta, \theta) \mathrm{d} \varrho=-\sum_{\varphi}(\zeta ; \eta, \theta) .
$$

Clearly,

$$
\left|\sum_{\varphi}(\zeta ; \eta, \theta)\right| \leqq n_{\infty}(\theta, \xi) \cdot \sup |\varphi| .
$$

Let us recall that $K_{\xi}$ is measurable $\left(H_{m-1}\right)$ by 2.6 in [10]. Noting that $\partial_{1} S \varphi(\varrho, \eta, \theta)$ is a continuous function on $(0, \infty) \times(0, \infty) \times \Gamma$ and taking into account that

$$
H_{m-1}\left(\Gamma \cap\left\{\theta ; \eta_{\infty}(\theta, \xi)=+\infty\right\}\right)=0
$$

we conclude that $\sum_{\varphi}(\zeta ; \eta, \theta)$ is measurable $\left(H_{m-1}\right)$ on $\Gamma$ and

$$
V_{\varphi}(\zeta ; \eta)=-\varphi(\zeta) H_{m-1}\left(K_{\xi}\right)-\int_{\Gamma} \mathrm{d} H_{m-1}(\theta) \int_{D^{*}} \partial_{1} S_{\varphi}(\varrho, \eta, \theta) \mathrm{d} \varrho
$$

is a Baire function of the variable $\eta>0$ satisfying the inequality

$$
\left|V_{\varphi}(\zeta ; \eta)\right| \leqq v_{\infty}(\xi) \sup |\varphi|
$$

Consider now the class $\mathscr{F}$ of all bounded Baire functions $f$ on $C$ for which $\sum_{f}(\zeta ; \eta, \theta)$ is integrable $\mathrm{d} H_{m-1}(\theta)$ over $\Gamma$ for each $\eta>0$ and $V_{f}(\zeta ; \eta)$ is a bounded Baire function of $\eta>0$. We have just seen that $\mathscr{F}$ contains restriction to $C$ of any $\varphi \in \mathscr{D}_{T_{2}}$. If $\left\{f_{k}\right\}_{k=1}^{\infty}$ is a sequence of elements of $\mathscr{F}$ with

$$
\lim _{k \rightarrow \infty} f_{k}=f
$$

such that, for suitable $K \in R^{1}$,

$$
\sup \left|f_{k}\right| \leqq K, \quad k \in N,
$$

then

$$
\lim _{k \rightarrow \infty} \sum_{f_{k}}(\zeta ; \eta, \theta)=\sum_{f}(\zeta ; \eta, \theta)
$$

and

$$
\left|\sum_{f_{k}}(\zeta ; \eta, \theta)\right| \leqq K n_{\infty}(\theta, \xi)
$$

for all $k \in N$. By the Lebesgue dominated convergence theorem also

$$
\lim _{k \rightarrow \infty} V_{f_{k}}(\zeta, \eta)=V_{f}(\zeta, \eta)
$$

Since

$$
\left|V_{f}(\zeta, \eta)\right| \leqq K v_{\infty}(\xi),
$$

we see that $f \in \mathscr{F}$. Consequently, $\mathscr{F}$ contains all bounded Baire functions on $C$ and the proof is complete. 
2.2. Corollary. Let $\zeta=[\xi, \tau] \in R_{T_{2}} \backslash R_{T_{1}}$ (see (9)) and denote by $d_{D}(\xi)$ the $m$ dimensional density of $D$ at $\xi$. Let $\varphi \in \mathscr{D}_{T_{2}}$ and define $\tilde{W} \varphi(\zeta)$ by 1.6. Then

$$
W \varphi(\zeta)=\tilde{W} \varphi(\zeta)-2^{m} \pi^{m / 2} d_{D}(\xi) \varphi(\zeta)
$$

Proof. Let us keep the notation from the above proof. According to 2.6 in [10]

$$
H_{m-1}\left(K_{\xi}\right)=d_{D}(\xi) 2 \pi^{m / 2} / \Gamma\left(\frac{1}{2} m\right) .
$$

Now it is sufficient to employ (33) and 1.6.

The following corollary was actually proved in the course of the proof of lemma 2.1.

2.3. Corollary. Let $\zeta=[\xi, \tau] \in R_{T_{2}} \backslash R_{T_{1}}$ and suppose that

$$
v_{\infty}(\xi)<+\infty \text {. }
$$

If $F$ is a bounded Baire function on $C$ then

$$
|W F(\zeta)| \leqq 2^{m-1} \Gamma\left(\frac{1}{2} m\right) v_{\infty}(\xi) \cdot \sup |F| .
$$

If $\left\{f_{k}\right\}$ is a pointwise convergent sequence of bounded Baire functions on $C$ such that for suitable $K \in R^{1}$,

and

$$
k \in N \Rightarrow\left|f_{k}\right| \leqq K
$$

$$
\lim _{k \rightarrow \infty} f_{k}=f
$$

then

$$
\lim _{k \rightarrow \infty} W f_{k}(\zeta)=W f(\zeta)
$$

Proof. The inequality (36) follows from the estimate

$$
\left|V_{F}(\zeta, \eta)\right| \leqq v_{\infty}(\xi) \sup |F|, \quad \eta>0 .
$$

Employing (37) with $F=f_{k}$ we get

$$
\left|V_{f_{k}}(\zeta, \eta)\right| \leqq K v_{\infty}(\xi), \quad \eta>0 .
$$

Now it is sufficient to use (34) and refer to the Lebesgue dominated convergence theorem.

2.4. Remark. Let us recall that a unit vector $\theta \in \Gamma$ is called the exterior normal of $D$ at $y \in R^{m}$ in the sense of H. FEDERER provided the symmetric difference of $D$ and the half-space

$$
R^{m} \cap\{x ;(x-y) \cdot \theta<0\}
$$


has $m$-dimensional density 0 at $y$. In what follows we shall put $n(y)=\theta$ if $\theta \in \Gamma$ is the exterior normal of $D$ at $y$ (which is easily seen to be uniquely determined) and we denote by $n(y)$ the zero vector if there is no exterior normal $\theta \in \Gamma$ at $y$ in the above mentioned sense. The set $\widehat{B}=R^{m} \cap\{y ;|n(y)| \neq 0\}$ will be termed the reduced boundary of $D$.

The following assertion is a consequence of proposition 2.10 in [10] and results of E. De Giorgi and H. Federer (see [2], [3] and 2.11. in [10]):

Proposition. Suppose there is an $(m+1)$-tuple of points $x^{1}, \ldots, x^{m+1} \in R^{m}$ in general position (i.e., not situated on a single hyperplane) such that

$$
\sum_{i=1}^{m+1} v_{\infty}\left(x^{i}\right)<\infty \text {. }
$$

Then $H_{m-1}(\hat{B})<+\infty$. If $w=\left[w_{1}, \ldots, w_{m}\right]$ is a vector-valued function with $m$ components $w_{j} \in \mathscr{D}$, then

$$
\int_{B} w(y) n(y) \mathrm{d} H_{m-1}(y)=\int_{D} \operatorname{div} w(x) \mathrm{d} x .
$$

In the rest of this chapter we shall always assume that

$$
\sup \left\{v_{\infty}(y) ; y \in B\right\}=V<\infty .
$$

As shown in theorem 2.13 in [10], (39) implies

$$
\sup \left\{v_{\infty}(x) ; x \in R^{m}\right\} \leqq V+H_{m-1}(\Gamma) .
$$

Consequently, (38) is valid for each $w$ satisfying the assumptions of the above proposition. This makes it possible to derive another useful integral representation for $W f$.

2.5. Lemma. If $f$ is a bounded Baire function on $C$ then, for each $\zeta=[\xi, \tau] \epsilon$ $\in R_{T_{2}} \backslash R_{T_{1}}$,

$$
W f(\zeta)=\int_{T_{1}}^{T_{2}} \mathrm{~d} t \int_{B} f(x, t) n(x) \cdot \hat{\nabla} G(x-\xi, t-\tau) \mathrm{d} H_{m-1}(x),
$$

where $n(x)$ is the exterior normal of $D$ at $x$ as defined in 2.4.

Proof. Fix $\zeta=[\xi, \tau] \in R_{T_{2}} \backslash R_{T_{1}}$ and suppose first that $f \in \mathscr{D}_{T_{2}}$ and $f$ vanishes in some neighbourhood of $\zeta$. Choose $\widetilde{G} \in \mathscr{D}$ so that

$$
\tilde{G}(z)=G(z-\zeta)
$$

for all $z$ in some neighborhood of spt $f$ and fix $t \in\left(T_{1}, T_{2}\right)$. Then (38) applies to $w=\left[w_{1}, \ldots, w_{m}\right]$ defined by

$$
w_{j}(y)=f(y, t) \cdot \partial_{j} \widetilde{G}(y, t)
$$


and we get for

$$
\begin{array}{r}
I(t)=\int_{B} f(y, t) n(y) \cdot \hat{\nabla} G(y-\xi, t-\tau) \mathrm{d} H_{m-1}(y)= \\
=\int_{D} \sum_{j=1}^{m}\left[\partial_{j} f(x, t) \partial_{j} \widetilde{G}(x, t)+f(x, t) \partial_{j}^{2} \widetilde{G}(x, t)\right] \mathrm{d} x .
\end{array}
$$

Noting that

$$
f \sum_{j=1}^{m} \partial_{j}^{2} \widetilde{G}=f \partial_{m+1} \widetilde{G}
$$

we obtain finally

$$
\begin{gathered}
\int_{T_{1}}^{T_{2}} I(t) \mathrm{d} t=\int_{T_{1}}^{T_{2}} \mathrm{~d} t \int_{D}\left[\hat{\nabla} f(x, t) . \hat{\nabla} G(x-\xi, t-\tau)-G(x-\xi, t-\tau) \partial_{m+1} f(x, t)\right] \mathrm{d} x= \\
=\tilde{W} f(\zeta) \leqq W f(\zeta) .
\end{gathered}
$$

Letting

$$
\mathscr{D}^{1}=\mathscr{D}_{T_{2}} \cap\{f ;|f| \leqq 1, \zeta \notin \operatorname{spt} f\}
$$

we conclude from proposition 1.8 that

$$
\begin{aligned}
& \int_{T_{1}}^{T_{2}} \mathrm{~d} t \int_{B}|n(x) \cdot \hat{\nabla} G(x-\xi, t-\tau)| \mathrm{d} H_{m-1}(x)= \\
= & \sup \left\{\tilde{W} f(\zeta) ; f \in \mathscr{D}^{1}\right\} \leqq 2^{m-1} \Gamma\left(\frac{1}{2} m\right) v_{\infty}(\xi)<\infty .
\end{aligned}
$$

We have so far verified that (41) is valid for $f \in \mathscr{D}_{T_{2}}$ vanishing near $\zeta$. Using corollary 2.3 and (42) one easily shows that (41) holds for an arbitrary bounded Baire function $f$ on $C$.

We shall now investigate the behavior of $W f(z)$ for $z$ approaching $C$. The following result is an analogue of theorem 2.15 in [10].

2.6. Theorem. Let

$$
D^{i}=R^{m} \cap\left\{x ; d_{D}(x)=i\right\}, \quad i=0,1 .
$$

Fix $\zeta=[\xi, \tau] \in C$ and suppose that $f$ is a bounded Baire function on $C$ such that

$$
\lim _{\substack{z \rightarrow \zeta \\ z \in C}} f(z)=\alpha .
$$

Then, for $i=0,1$,

$$
\left(z \in D^{i} \times\left\langle T_{1}, T_{2}\right), z \rightarrow \zeta\right) \Rightarrow W f(z) \rightarrow W f(\zeta)+\alpha\left[d_{D}(\xi)-i\right] 2^{m} \pi^{m / 2}
$$


Proof. We shall assume that $T_{2}=+\infty$ (for we may always extend $f$ to $B \times$ $\times\left\langle T_{1},+\infty\right)$ defining $f(y, t)=0$ for $t \in\left\langle T_{2},+\infty\right)$ and $\left.y \in B\right)$. We are going to evaluate $W f(\zeta)$ for any $\zeta=[\xi, \tau] \in R^{m} \times\left\langle T_{1},+\infty\right)$ assuming that

$$
f=1 \text { on } C=B \times\left\langle T_{1},+\infty\right) \text {. }
$$

Define $K_{\xi}$ as in the proof of lemma 2.1. Fix $\theta \in \Gamma$ with $n_{\infty}(\theta, \xi)<+\infty$ and let

$$
D_{\theta}=\{\varrho ; \varrho>0, \xi+\varrho \theta \in D\} .
$$

If $\varrho_{1}<\ldots<\varrho_{n}$ are all the hits of $(0,+\infty)$ on $D_{\theta}$, then $(30)$ holds. Besides that,

$$
\begin{aligned}
& \theta \in K_{\xi} \Rightarrow s\left(\varrho_{1} ; \xi, \theta\right)=-1, \\
& \theta \in \Gamma \backslash K_{\xi} \Rightarrow s\left(\varrho_{1} ; \xi, \theta\right)=1
\end{aligned}
$$

and $s\left(\varrho_{n} ; \xi, \theta\right)=-1$ or $s\left(\varrho_{n} ; \xi, \theta\right)=1$ according as $D$ is bounded or not. We thus conclude for bounded $D$

$$
\begin{gathered}
\left(\theta \in K_{\xi}, \eta>0\right) \Rightarrow \sum_{f}(\zeta ; \eta, \theta)=-1, \\
\left(\theta \in \Gamma \backslash K_{\xi}, \eta>0\right) \Rightarrow \sum_{f}(\zeta, \eta, \theta)=0,
\end{gathered}
$$

while for unbounded $D$

$$
\begin{array}{lll}
\theta \in K_{\xi} \quad \Rightarrow \sum_{f}(\zeta ; \eta, \theta)=0 & \text { for all } & \eta>0, \\
\theta \in \Gamma \backslash K_{\xi} \Rightarrow \sum_{f}(\zeta ; \eta, \theta)=1 & \text { for all } & \eta>0 .
\end{array}
$$

Employing (35) we obtain for bounded $D$

$$
W f(\zeta)=-2^{m} d_{D}(\xi) \pi^{m / 2},
$$

while for unbounded $D$

$$
W f(\zeta)=+2^{m}\left[1-d_{D}(\xi)\right] \pi^{m / 2}
$$

Since $\xi \in R^{m}$ was arbitrary, we see that (44) holds with $\alpha=1$ for $f$ satisfying (45). It remains to verify (44) provided (43) holds with $\alpha=0$. We may clearly assume that $f(\zeta)=0$, too. Then, for any $\varepsilon>0$, there is a decomposition $f=f_{\varepsilon}+g_{\varepsilon}$ such that $g_{\varepsilon}$ is a bounded Baire function vanishing in some nieghborhood of $\zeta$ in $C$ and $\left|f_{\varepsilon}\right| \leqq \varepsilon$ on $C$. It follows from lemma 2.5 that

$$
\lim _{z \rightarrow \zeta} W g_{\varepsilon}(z)=W g_{\varepsilon}(\zeta)
$$

On the other hand, (36) together with (40) imply

$$
\left|W f_{\varepsilon}(z)\right| \leqq 2^{m-1} \varepsilon\left[\Gamma\left(\frac{1}{2} m\right) V+2 \pi^{m / 2}\right]
$$


for all $z \in R^{m} \times\left\langle T_{1},+\infty\right)$. Since $\varepsilon>0$ can be chosen as small as we want, we conclude that in this case

$$
W f(\zeta)=\lim _{z \rightarrow \zeta} W f(z), \quad z \in R^{m} \times\left\langle T_{1},+\infty\right),
$$

and the proof is complete.

2.7. Definition. Let $-\infty<T_{1}<T_{2}<+\infty$ and denote by $\mathscr{B}=\mathscr{B}\left(T_{1}, T_{2}\right)$ the Banach space of all continuous functions on $B \times\left\langle T_{1}, T_{2}\right\rangle$ vanishing on $B \times\left\{T_{2}\right\}$, equipped with the supremum norm. Given $f \in \mathscr{B}$ and $\alpha \in R^{1}$ define $W_{\alpha} f$ on $B \times$ $\times\left\langle T_{1}, T_{2}\right\rangle$ letting for $\xi \in B$

$$
\begin{gathered}
W_{\alpha} f\left(\xi, T_{2}\right)=0, \\
W_{\alpha} f(\xi, \tau)=W f(\xi, \tau)+2^{m} \pi^{m / 2}\left[d_{D}(\xi)-\alpha\right] f(\xi, \tau), \quad T_{1} \leqq \tau<T_{2} .
\end{gathered}
$$

2.8. Lemma. Fix $\alpha \in R^{1}$. Then

$$
f \in \mathscr{B} \Rightarrow W_{\alpha} f \in \mathscr{B} .
$$

The operator $W_{\alpha}: f \rightarrow W_{\alpha} f$ is bounded on $\mathscr{B}$ and

$$
\left\|W_{\alpha}\right\| \leqq\left[V \Gamma\left(\frac{1}{2} m\right)+(1+|\alpha|) 2 \pi^{m / 2}\right] 2^{m-1} .
$$

If $I^{\prime}$ stands for the identity operator on $\mathscr{B}^{\prime}$, then the operator

$$
H_{\alpha}=H-\alpha 2^{m} \pi^{m / 2} I^{\prime}
$$

(acting on $\mathscr{B}^{\prime}$ ) is dual to $W_{\alpha}$.

Proof. Fix $f \in \mathscr{B}$ and define $F$ on $B \times\left\langle T_{1},+\infty\right)$ so that $F=f$ on $B \times\left\langle T_{1}, T_{2}\right\rangle$, $F=0$ on $B \times\left(T_{2},+\infty\right)$. Then $F$ is continuous on

$$
\left.C_{\infty}=B \times<T_{1},+\infty\right)
$$

According to theorem 2.6 (where now $C$ is replaced by $C_{\infty}$ ),

$$
L(\xi, \tau)=\lim W F(x, t)\left(x \in D, x \rightarrow \xi ; t>\Gamma_{1}, t \rightarrow \tau\right)
$$

is defined for $[\xi, \tau] \in C_{\infty}$ and

$$
W F(\xi, \tau)+2^{m} \pi^{m / 2} d_{D}(\xi) F(\xi, \tau)=2^{m} \pi^{m / 2} F(\xi, \tau)+L(\xi, \tau)
$$

is a continuous function of $[\xi, \tau] \in C_{\infty}$ vanishing on $B \times\left\langle T_{2},+\infty\right)$. Noting that, for $[\xi, \tau] \in B \times\left\langle T_{1}, T_{2}\right\rangle=\mathrm{cl} C, W_{\alpha} f(\xi, \tau)$ coincides with

$$
W F(\xi, \tau)+2^{m} \pi^{m / 2}\left[d_{D}(\xi)-\alpha\right] F(\xi, \tau),
$$


we conclude that $W_{\alpha} f \in \mathscr{B}$. The estimate (46) follows at once from the definition of $W_{\alpha}$ and (36).

If $F$ is a function with domain containing $\operatorname{cl} C$ such that $f=F \mid \operatorname{cl} C$ (= the restriction of $F$ to $\mathrm{cl} C$ ) belongs to $\mathscr{B}$, we agree to use $W_{\alpha} F$ to denote $W_{\alpha} f$.

Consider now $\varphi \in \mathscr{D}_{T_{2}}$. It follows from 2.7 and 2.2 that, for $\zeta \in C$,

$$
W_{\alpha} \varphi(\zeta)=\tilde{W} \varphi(\zeta)-2^{m} \pi^{m / 2} \alpha \varphi(\zeta) .
$$

Employing (20) and (27) we conclude that

$$
\left\langle W_{\alpha} \varphi, \mu\right\rangle=\left\langle\varphi, H_{\alpha} \mu\right\rangle, \quad \mu \in \mathscr{B}^{\prime}, \quad \varphi \in \mathscr{D}_{T_{2}} .
$$

If $f \in \mathscr{B}$, then there is a sequence $\varphi_{n} \in \mathscr{D}_{T_{2}}(n=1,2, \ldots)$ such that $\varphi_{n} \rightarrow f$ uniformly on $B \times\left\langle T_{1}, T_{2}\right\rangle$ as $n \rightarrow \infty$. Hence it follows that

$$
\left(\mu \in \mathscr{B}^{\prime}, f \in \mathscr{B}\right) \Rightarrow\left\langle W_{\alpha} f, \mu\right\rangle=\left\langle f, H_{\alpha} \mu\right\rangle
$$

and the proof is complete.

Remark. Let us denote by I the identity operator acting on $\mathscr{B}$. It follows from 2.8 that the operator $H$ is dual to

$$
\alpha 2^{m} \pi^{m / 2} I+W_{\alpha} .
$$

Accordingly, the following simple result appears to be useful in connection with investigations of the range of $H$.

2.9. Proposition. Fix $\alpha, \beta \in R^{1}$ and denote by $\mathscr{B}_{\alpha \beta}$ the class of all $f \in \mathscr{B}$ satisfying

$$
\left(\beta I+W_{\alpha}\right) f=0 .
$$

Then $\mathscr{B}_{\alpha \beta}$ is a subspace of $\mathscr{B}$ which is either trivial (i.e., the function vanishing identically on $\mathrm{cl} C$ is the only element of $\mathscr{B}_{\alpha \beta}$ ) or infinite dimensional.

Proof. For $\varepsilon \geqq 0$ and $f \in \mathscr{B}$ define $T^{\varepsilon} f$ as follows. Given $\xi \in B$, let $\mathscr{J}=\left\langle T_{1}, T_{2}\right\rangle$ and put

$$
\begin{aligned}
& T^{\varepsilon} f(\xi, t)=0 \text { for } t \in \mathscr{J} \cap\left\langle T_{2}-\varepsilon,+\infty\right), \\
& T^{\varepsilon} f(\xi, t)=f(\xi, t+\varepsilon) \text { for } t \in \mathscr{J} \cap\left(-\infty, T_{2}-\varepsilon\right) .
\end{aligned}
$$

Clearly, $T^{\varepsilon}(\mathscr{B}) \subset \mathscr{B}$ for each $\varepsilon>0$. It follows easily from the definition of $W_{\alpha}$ and 2.5 (note also that $G(z)=0$ for $z \in \mathrm{cl} R_{0}$ ) that

$$
W_{\alpha} T^{\varepsilon} f=T^{\varepsilon} W_{\alpha} f, \quad f \in \mathscr{B} .
$$

Consequently, also $\mathscr{B}_{\alpha \beta}$ is translation invariant in the sense that $T^{\varepsilon}\left(\mathscr{B}_{\alpha \beta}\right) \subset \mathscr{B}_{\alpha \beta}$ for each $\varepsilon>0$. Now it is sufficient to employ the following elementary lemma: 
Let $g$ be a continuous function on $\mathscr{J}, g\left(T_{2}\right)=0$, and define for each $\varepsilon \geqq 0$

$$
\begin{aligned}
& T^{\varepsilon} g(t)=0 \text { for } t \in \mathscr{J} \cap\left\langle T_{2}-\varepsilon,+\infty\right), \\
& T^{\varepsilon} g(t)=g(t+\varepsilon) \text { for } t \in \mathscr{J} \cap\left(-\infty, T_{2}-\varepsilon\right) .
\end{aligned}
$$

If

$$
\tau=\inf \{t ; t \in \mathscr{J}, g(t)=0\}>T_{1},
$$

then, for each choice of $n \in N$ and $\varepsilon>0$ with

$$
T_{1}+n \varepsilon<\tau,
$$

the functions in $\left\{T^{(k-1) \varepsilon} g\right\}_{k=1}^{n}$ are linearly independent.

Indeed, for $k=1, \ldots, n, T^{(k-1) \varepsilon} g$ does not vanish identically on

$$
\mathscr{J}_{k \varepsilon}=\langle\tau-k \varepsilon, \tau-(k-1) \varepsilon\rangle,
$$

while all $T^{j \varepsilon} g$ with $j \geqq k$ do vanish on $\mathscr{J}_{k \varepsilon}$. The rest is obvious.

\section{CHAPTER 3}

Unless the contrary is explicitly stated, in this chapter we always assume that

$$
\sup \left\{v_{\infty}(\xi) ; \xi \in B\right\}=V<+\infty \text {. }
$$

We proceed to investigate the dual equations

$$
H \mu=v\left(\text { over } \mathscr{B}^{\prime}\right), \quad W_{0} f=g(\text { over } \mathscr{B})
$$

associated with the Fourier problem. The methods usually used when $B$ is a sufficiently smooth hypersurface are no longer applicable under the general assumption (47). (Under appropriate smoothness assumptions on $B$ the resolvent of the resulting integral equation can be evaluated in the form of a series; cf. [20], where also further references to the work of E. Holmgren, E. Levi, M. Gevrey, H. Müntz, S. G. Michlin, A. N. Tichonov may be found. See also [7], [8], [11], [13], [14], [17].)

We consider the decompositions

$$
H=2^{m} \pi^{m / 2} \alpha I^{\prime}+H_{\alpha}, \quad W_{0}=2^{m} \pi^{m / 2} \alpha I+W_{\alpha}
$$

and evaluate the Fredholm radius of $W_{\alpha}$, which is the reciprocal of the quantity

$$
\omega W_{\alpha}=\inf _{Q}\|W-Q\|
$$

where $Q$ ranges over all compact operators acting on $\mathscr{B}$. It appears that $\omega W_{\alpha}$ can be expressed in geometric terms connected with $D$. This makes it possible to find the 
optimal value $\gamma$ of the parameter $\alpha$ in dependence on the shape of $D$ and establish conditions on $D$ guaranteeing

$$
\omega W_{\gamma}<2^{m} \pi^{m / 2}|\gamma|
$$

The Riesz-Schauder theory together with proposition 2.9 then yield the desired result concerning the Fourier problem.

Remark. We shall see that the optimal value of the parameter $\alpha$, for which $\omega W_{\alpha} /|\alpha|$ attains its minimum, equals $\frac{1}{2}$ if $d_{D}(x)=\frac{1}{2}$ for all $x \in B$. This naturally occurs if $B$ is a smooth hypersurface. It is interesting to observe that under the assumption (47) the optimal value of the parameter may be different from $\frac{1}{2}$ (see 3.9 below).

It should be noted here that already J. RADON considered the quantity corresponding to $\omega W_{\alpha}$ for special choice of $\alpha$ in his investigations of the logarithmic potential; he evaluated it for plane domains bounded by curves with bounded rotation (see $[21],[22])$. Compare also [10], [24] treating boundary value problems for Newtonian potentials in $n$-space.

3.1. Notation. Throughout this chapter we assume that $-\infty<T_{1}<T_{2}<$ $<+\infty$. Given $\varepsilon, \delta>0$ and $\zeta=[\xi, \tau] \in B \times\left\langle T_{1}, T_{2}\right\rangle=\mathrm{cl} C$, we denote by $\chi_{\zeta}^{\varepsilon \delta}$ the characteristic function of

$$
M_{\zeta}(\varepsilon, \delta)=R^{m+1} \backslash\left[\Omega_{\varepsilon}(\xi) \times(\tau-\delta, \tau+\delta)\right] .
$$

$\widehat{B}$ and $n$ will denote the reduced boundary and exterior normal of $D$, respectively, as defined in 2.4. For $0<r<\varepsilon$ put

$$
q_{\varepsilon}(r)=\sup _{x \in B} H_{m-1}\left\{\widehat{B} \cap\left[\Omega_{\varepsilon+r}(x) \backslash \Omega_{\varepsilon-r}(x)\right]\right\} .
$$

We define for each bounded Baire function $f$ on $\mathrm{cl} C$ and $\zeta=[\xi, \tau] \in \mathrm{cl} C$

$$
W^{\varepsilon \delta} f(\zeta)=\int_{T_{1}}^{T_{2}} \mathrm{~d} t \int_{B} \chi_{\zeta}^{\varepsilon \delta}(x, t) f(x, t) n(x) \cdot \hat{\nabla} G(x-\xi, t-\tau) \mathrm{d} H_{m-1}(x) .
$$

3.2. Lemma. Fix $\varepsilon, \delta>0$. Then there is a positive constant $c \in R^{1}$ such that

$$
\left|W^{\varepsilon \delta} f(\zeta)-W^{\varepsilon \delta} f(\bar{\zeta})\right| \leqq c\left[q_{\varepsilon}(|\zeta-\bar{\zeta}|)+|\zeta-\bar{\zeta}|\right]
$$

for each Baire function $f$ satisfying

$$
\sup \{|f(z)| ; z \in \operatorname{cl} C\} \leqq 1
$$

and each couple of points $\zeta=[\xi, \tau], \bar{\zeta}=[\bar{\xi}, \bar{\tau}]$ in cl $C$ satisfying

$$
|\xi-\bar{\xi}|<\frac{1}{2} \varepsilon, \quad|\tau-\bar{\tau}|<\frac{1}{2} \delta \text {. }
$$


Proof. It is easy to see that there is a $c_{1} \in R^{1}$ such that

$$
|\hat{\nabla} G| \leqq c_{1}
$$

in

$$
M_{0}\left(\frac{1}{2} \varepsilon, \frac{1}{2} \delta\right)=\left\{z ;\left|z_{m+1}\right|>\frac{1}{2} \delta\right\} \cup\left\{z ;|\hat{z}|>\frac{1}{2} \varepsilon\right\}
$$

and

$$
|\hat{\nabla} G(u)-\hat{\nabla} G(\bar{u})| \leqq c_{1}|u-\bar{u}|
$$

for each couple of points $u, \bar{u}$ in $M_{0}\left(\frac{1}{2} \varepsilon, \frac{1}{2} \delta\right)$. Consider now $\zeta=[\xi, \tau], \bar{\zeta}=[\bar{\zeta}, \bar{\tau}] \epsilon$ $\in R^{m+1}$ satisfying (51) and suppose that $f$ is a Baire function on $\mathrm{cl} C$ satisfying (50). Writing $z=[x, t]$,

$$
\begin{aligned}
& J_{1}=\int_{T_{1}}^{T_{2}} \mathrm{~d} t \int_{B} \chi_{\zeta}^{\varepsilon \delta}(\dot{z}) f(z) n(x) \cdot[\hat{\nabla} G(z-\zeta)-\hat{\nabla} G(z-\bar{\zeta})] \mathrm{d} H_{m-1}(x), \\
& J_{2}=\int_{T_{1}}^{T_{2}} \mathrm{~d} t \int_{B}\left[\chi_{\zeta}^{\varepsilon \delta}(z)-\chi_{\zeta}^{\varepsilon \delta}(z)\right] f(z) n(x) \cdot \hat{\nabla} G(z-\bar{\zeta}) \mathrm{d} H_{m-1}(x),
\end{aligned}
$$

we have

$$
\left|W^{\varepsilon \delta} f(\zeta)-W^{\varepsilon \delta} f(\bar{\zeta})\right| \leqq\left|J_{1}\right|+\left|J_{2}\right|
$$

If $z$ is in $M_{\zeta}(\varepsilon, \delta)$ then, in view of (51), both $z-\zeta$ and $z-\bar{\zeta}$ belong to $M_{0}\left(\frac{1}{2} \varepsilon, \frac{1}{2} \delta\right)$, so that

$$
\left|J_{1}\right| \leqq c_{1}|\zeta-\bar{\zeta}| H_{m-1}(\widehat{B})\left(T_{2}-T_{1}\right)
$$

Put

$$
R=\varepsilon+|\xi-\xi|, \quad r=\varepsilon-|\xi-\xi| .
$$

Then the symmetric difference of $M_{\zeta}(\varepsilon, \delta)$ and $M_{\xi}(\varepsilon, \delta)$ is contained in the union of

and

$$
\left[\Omega_{R}(\xi) \backslash \Omega_{r}(\xi)\right] \times R^{1}
$$

$$
R^{m} \times\{t ; \delta-|\tau-\bar{\tau}| \leqq|t-\tau|<\delta+|\tau-\bar{\tau}|\} .
$$

Hence it follows

$$
\left|J_{2}\right| \leqq c_{1} q_{\varepsilon}(|\xi-\bar{\xi}|)\left(T_{2}-T_{1}\right)+4 c_{1} H_{m-1}(\hat{B})|\tau-\bar{\tau}| .
$$

Combining (52), (53) and (54) we get (49) with

$$
c=\max \left\{c_{1}\left(T_{2}-T_{1}\right), c_{1} H_{m-1}(\hat{B})\left[\left(T_{2}-T_{1}\right)+4\right]\right\} .
$$


3.3. Lemma. Given $\varepsilon, \delta>0$ and $\zeta=[\xi, \tau] \in R^{m+1}$, denote by $\chi_{\zeta}^{\varepsilon \delta}$ the characteristic function of

and define

$$
\Omega_{\varepsilon}(\xi) \times(\tau, \tau+\delta)
$$

$$
v^{\varepsilon \delta}(\zeta)=\int_{T_{1}}^{T_{2}} \mathrm{~d} t \int_{B} x_{\zeta}^{\varepsilon \delta}(x, t)|n(x) \cdot \hat{\nabla} G(x-\xi, t-\tau)| \mathrm{d} H_{m-1}(x) .
$$

If $M \subset C$ is dense in $C$, then

$$
\begin{aligned}
\omega W_{\alpha} & \leqq \sup _{\zeta \in M}\left\{2^{m} \pi^{m / 2}\left|d_{D}(\hat{\zeta})-\alpha\right|+v^{\varepsilon \delta}(\zeta)\right\}= \\
& =\sup _{\zeta \in \zeta}\left\{2^{m} \pi^{m / 2}\left|d_{D}(\hat{\zeta})-\alpha\right|+v^{\varepsilon \delta}(\zeta)\right\}
\end{aligned}
$$

for all $\alpha \in R^{1}$ and $\varepsilon, \delta>0$.

Proof. Fix $\alpha \in R^{1}$ and $\varepsilon, \delta>0$. Noting that $v^{\varepsilon \delta}(\zeta)$ is a non-decreasing function of the variable $\varepsilon>0$, we may assume for the proof of (55) that

$$
x \in B \Rightarrow H_{m-1}\left[\hat{B} \cap \text { fr } \Omega_{\varepsilon}(x)\right]=0 .
$$

Indeed, the set of those $\varepsilon>0$ for which (56) is violated is at most countable, because

$$
\sum_{i=1}^{n} H_{m-1}\left(\hat{B} \cap S_{i}\right) \leqq H_{m-1}(\hat{B})<+\infty
$$

for each choice of spheres $S_{i}=\operatorname{fr} \Omega_{\varepsilon_{i}}\left(x^{i}\right)$ with mutually different radii $\varepsilon_{1}>\ldots>\varepsilon_{n}$ and arbitrary $n \in N$.

Defining $q_{\varepsilon}$ by (48) we conclude from (56) that

$$
\lim _{r \rightarrow 0+} q_{\varepsilon}(r)=0 \text {. }
$$

It follows from lemma 3.2 that all the functions in

$$
\left\{W^{\varepsilon \delta} f ; f \in \mathscr{B},\|f\| \leqq 1\right\}
$$

are equicontinuous on $\mathrm{cl} C$. Employing (36) one easily sees that (57) is contained in $\mathscr{B} \cap\left\{g ;\|g\| \leqq 2^{m-1} \Gamma\left(\frac{1}{2} m\right) V\right\}$ (see also (47)). Consequently, $W^{\varepsilon \delta}: f \rightarrow W^{\varepsilon \delta}$ is a compact operator on $\mathscr{B}$ and

$$
\omega W_{\alpha} \leqq\left\|W_{\alpha}-W^{\varepsilon \delta}\right\|
$$

Noting that $G$ vanishes on $\operatorname{cl} R_{0}$ we get from (41) for $f \in \mathscr{B}$ and $\zeta=[\xi, \tau] \in \operatorname{cl} C$

$$
\begin{gathered}
\left(W_{\alpha}-W^{\varepsilon \delta}\right) f(\zeta)=2^{m} \pi^{m / 2}\left[d_{D}(\xi)-\alpha\right] f(\zeta)+ \\
+\int_{T_{1}}^{T_{2}} \mathrm{~d} t \int_{B} f(x, t) x_{\zeta}^{\varepsilon \delta}(x, t) n(x) \cdot \hat{\nabla} G(x-\xi, t-\tau) \mathrm{d} H_{m-1}(x),
\end{gathered}
$$


whence it follows that

$$
\begin{gathered}
2^{m} \pi^{m / 2}\left|d_{D}(\hat{\zeta})-\alpha\right|+v^{\varepsilon \delta}(\zeta)= \\
=\sup \left\{\left(W_{\alpha}-W^{\varepsilon \delta}\right) f(\zeta) ; f \in \mathscr{B},\|f\| \leqq 1\right\}
\end{gathered}
$$

is a lower-semicontinuous function of $\zeta \in \mathrm{cl} C$ and

$$
\begin{gathered}
\left\|W_{\alpha}-W^{\varepsilon \delta}\right\|=\sup _{\zeta \in M}\left\{2^{m} \pi^{m / 2}\left|d_{D}(\hat{\zeta})-\alpha\right|+v^{\varepsilon \delta}(\zeta)\right\}= \\
=\sup _{\zeta \in C}\left\{2^{m} \pi^{m / 2}\left|d_{D}(\hat{\zeta})-\alpha\right|+v^{\varepsilon \delta}(\zeta)\right\} .
\end{gathered}
$$

This together with (58) completes the proof.

The following slight modification of a known result due to J. RADON will be needed below.

3.4. Lemma. If $Q$ is a compact operator on $\mathscr{B}$ then, for every $\varepsilon>0$, there exist $f_{1}, \ldots, f_{s} \in \mathscr{B}$ and $\mu_{1}, \ldots, \mu_{s} \in \mathscr{B}^{\prime}$ such that the operator

$$
Q_{\varepsilon}: f \rightarrow \sum_{i=1}^{s}\left\langle f, \mu_{i}\right\rangle f_{i}, \quad f \in \mathscr{B},
$$

satisfies

$$
\left\|Q-Q_{\varepsilon}\right\| \leqq \varepsilon .
$$

Proof. For $z \in \operatorname{cl} C$ define $\Phi(z) \in \mathscr{B}^{\prime}$ by

Accordingly,

$$
\langle f, \Phi(z)\rangle=Q f(z), \quad f \in \mathscr{B} .
$$

$$
\left(z \in \operatorname{cl} C, z_{m+1}=T_{2}\right) \Rightarrow\|\Phi(z)\|=0 .
$$

Since $Q$ is a compact operator on $\mathscr{B}, \Phi$ is a continuous map on cl $B$ to $\mathscr{B}^{\prime}$ (compare [22], chap. $\mathrm{V}, \mathrm{n}^{\circ} 90$, p. 218). Consequently, we may fix $T \in\left(T_{1}, T_{2}\right)$ such that

$$
\text { , }\left(z \in C, z_{m+1}>T\right) \Rightarrow\|\Phi(z)\|<\varepsilon .
$$

Further choose open sets $U_{1}, \ldots, U_{s}$ with

$$
C \cap \operatorname{cl} R_{T} \subset \bigcup_{i=1}^{s} U_{i} \subset R_{T_{2}}
$$

such that, for $i=1, \ldots, s$,

$$
\left(z, \bar{z} \in C \cap U_{i}\right) \Rightarrow\|\Phi(z)-\Phi(\bar{z})\|<\varepsilon .
$$

Put

$$
U_{0}=R^{m+1} \cap\left\{z ; z_{m+1}>T\right\},
$$


so that

$$
U_{0}, U_{1}, \ldots, U_{s}
$$

is an open covering of $\mathrm{cl} C$. Associate with (61) the decomposition of unity formed by continuous non-negative functions $f_{0}, f_{1}, \ldots, f_{s}$ on $\mathrm{cl} C$ such that

$$
\text { spt } f_{j} \subset U_{j}(0 \leqq j \leqq s), \sum_{j=0}^{s} f_{j}=1 \quad \text { on } \quad \operatorname{cl} C .
$$

Fix $z^{i} \in C \cap U_{i}$, put $\mu_{i}=\Phi\left(z^{i}\right)(1 \leqq i \leqq s)$ and define $Q_{\varepsilon}$ by (59). Consider now an arbitrary $f \in \mathscr{B}$ with $\|f\| \leqq 1$. We have

$$
\left(Q-Q_{\varepsilon}\right) f(z)=\langle f, \Phi(z)\rangle f_{0}(z)+\sum_{i=1}^{s}\left\langle f, \Phi(z)-\Phi\left(z^{i}\right)\right\rangle f_{i}(z) .
$$

Since $f_{0}$ vanishes outside $U_{0}$ and $\|\Phi(z)\|<\varepsilon$ for $z \in U_{0} \cap$ cl. $C$, we have

$$
\left|\langle f, \Phi(z)\rangle f_{0}(z)\right| \leqq \varepsilon f_{0}(z) .
$$

Note that $f_{1}, \ldots, f_{s}$ vanish outside $\bigcup_{i=1}^{s} U_{i}$, while

for $z$ in

$$
\left\|\Phi(z)-\Phi\left(z^{i}\right)\right\|<\varepsilon
$$

$$
U_{i} \cap \operatorname{cl} C \supset\left\{z ; f_{i}(z) \neq 0\right\}, \quad i=1, \ldots, s .
$$

Consequently,

$$
\left|\sum_{i=1}^{s}\left\langle f, \Phi(z)-\Phi\left(z^{i}\right)\right\rangle f_{i}(z)\right| \leqq \varepsilon\left[1-f_{0}(z)\right] .
$$

Combining (64), (63) and (62) we get (60).

3.5. Lemma. Let us keep the notation from lemma 3.3. Then

$$
\omega W_{\alpha} \geqq \lim _{\varepsilon, \delta \rightarrow 0+} \sup _{\zeta \in C}\left\{2^{m} \pi^{m / 2}\left|d_{D}(\hat{\zeta})-\alpha\right|+v^{\varepsilon \delta}(\zeta)\right\}
$$

for every $\alpha \in R^{1}$.

Proof. Fix $\alpha \in R^{1}$ and let

$$
k>\omega W_{\alpha} .
$$

According to lemma 3.4 , there are $f_{1}, \ldots, f_{s} \in \mathscr{B}$ and $\mu_{1}, \ldots, \mu_{s} \in \mathscr{B}^{\prime}$ such that the operator

$$
Q: f \rightarrow \sum_{i=1}^{s}\left\langle f, \mu_{i}\right\rangle f_{i}, f \in \mathscr{B},
$$


satisfies

$$
k>\left\|W_{\alpha}-Q\right\|
$$

Writing $c_{M}$ for the characteristic function of $M$ we associate with each $\zeta=[\xi, \tau] \in C$ the measure $v_{\zeta}$ defined on the system of Borel sets $M \subset R^{m+1}$ by

$$
v_{\zeta}(M)=\int_{T_{1}}^{T_{2}} \mathrm{~d} t \int_{B} c_{M}(x, t) n(x) \cdot \hat{\nabla} G(x-\xi, t-\tau) \mathrm{d} H_{m-1}(x) .
$$

Clearly, $v_{\zeta} \in \mathscr{B}^{\prime}$. Denoting by $\delta_{\zeta}$ the Dirac measure (= unit point mass) concentrated at $\zeta$ we have by $(41)$

$$
W_{\alpha} f(\zeta)=\left\langle f, v_{\zeta}+2^{m} \pi^{m / 2}\left[d_{D}(\hat{\zeta})-\alpha\right] \delta_{\zeta}\right\rangle, \quad \zeta \in C, \quad f \in \mathscr{B}
$$

Let

$$
C_{0}=C \cap\left\{\zeta ; \sum_{i=1}^{s}\left|\mu_{i}\right|(\{\zeta\})=0\right\}
$$

Clearly, $C \backslash C_{0}$ is at most countable. Consequently,

$$
\left|v_{\zeta}\right|\left(C \backslash C_{0}\right)=0=\left|v_{\zeta}\right|(\{\zeta\}) \text { for every } \zeta \in C .
$$

For $i=1, \ldots, s$ consider the decomposition

$$
\mu_{i}=\mu_{i}^{1}+\mu_{i}^{2}
$$

where

$$
\begin{gathered}
\mu_{i}^{1}, \mu_{i}^{2} \in \mathscr{B}^{\prime}, \\
z \in R^{m+1} \Rightarrow\left|\mu_{i}^{1}\right|(\{z\})=0, \\
\left|\mu_{i}^{2}\right|\left(C_{0}\right)=0 .
\end{gathered}
$$

In view of $(66)-(69)$ we have then for each $\zeta \in C_{0}$

$$
\begin{gathered}
k>\left\|W_{\alpha}-Q\right\|>\left\|v_{\zeta}+2^{m} \pi^{m / 2}\left[d_{D}(\hat{\zeta})-\alpha\right] \delta_{\zeta}-\sum_{i=1}^{s} f_{i}(\zeta) \mu_{i}\right\|= \\
=\left\|v_{\zeta}-\sum_{i=1}^{s} f_{i}(\zeta) \mu_{i}^{1}\right\|+2^{m} \pi^{m / 2}\left|d_{D}(\hat{\zeta})-\alpha\right|+ \\
\therefore+\left\|\sum_{i=1}^{s} f_{i}(\zeta) \mu_{i}^{2}\right\| \geqq\left\|v_{\zeta}-\sum_{i=1}^{s} f_{i}(\zeta) \mu_{i}^{1}\right\|+2^{m} \pi^{m / 2}\left|d_{D}(\hat{\zeta})-\alpha\right| .
\end{gathered}
$$

Put

$$
K=\max \left\{\left|f_{i}(z)\right| ; z \in \mathrm{cl} C, 1 \leqq i \leqq s\right\}
$$


and define $x_{\zeta}^{\varepsilon \delta}$ as in lemma 3.3. For $\mu \in \mathscr{B}^{\prime}, x_{\zeta}^{\varepsilon \delta} \mu \in \mathscr{B}^{\prime}$ is defined by $\left\langle f, x_{\zeta}^{\varepsilon \delta} \mu\right\rangle=$ $=\left\langle f x_{\zeta}^{\varepsilon \delta}, \mu\right\rangle(f \in \mathscr{B})$, as usual. Then

$$
\begin{gathered}
\left\|v_{\zeta}-\sum_{i=1}^{s} f_{i}(\zeta) \mu_{i}\right\| \geqq\left\|x_{\zeta}^{\varepsilon \delta}\left[v_{\zeta}-\sum_{i=1}^{s} f_{i}(\zeta) \mu_{i}^{1}\right]\right\| \geqq \\
\geqq\left\|x_{\zeta}^{\varepsilon \delta} v_{\zeta}\right\|-K \sum_{i=1}^{s} \sup \left\{\left\|x_{\zeta}^{\varepsilon \delta} \mu_{i}^{1}\right\| ; \zeta \in \operatorname{cl} C\right\},
\end{gathered}
$$

whence we conclude by $(70)$

$$
k>\sup _{\zeta \in C_{0}}\left\{2^{m} \pi^{m / 2}\left|d_{D}(\hat{\zeta})-\alpha\right|+\left\|x_{\zeta}^{\varepsilon \delta} v_{\zeta}\right\|\right\}-K \sum_{i=1}^{s} \sup \left\{\left\|x_{\zeta}^{\varepsilon \delta} \mu_{i}^{1}\right\| ; \zeta \in \operatorname{cl} C\right\} .
$$

Noting that $C_{0}$ is dense in $C$ and $\left\|x_{\zeta}^{\varepsilon \delta} v_{\zeta}\right\|=v^{\varepsilon \delta}(\zeta)$ as introduced in lemma 3.3, we get by (55)

$$
\sup _{\zeta \in C_{0}}\left\{2^{m} \pi^{m / 2}\left|d_{D}(\hat{\zeta})-\alpha\right|+\left\|x_{\zeta}^{\varepsilon \delta} v_{\zeta}\right\|\right\}=\sup _{\zeta \in C}\left\{2^{m} \pi^{m / 2}\left|d_{D}(\hat{\zeta})-\alpha\right|+v^{\varepsilon \delta}(\zeta)\right\} .
$$

Since cl $C$ is compact, (68) implies

$$
\lim _{\varepsilon, \delta \rightarrow 0+} \sum_{i=1}^{s} \sup \left\{\left\|\chi_{\zeta}^{\varepsilon \delta} \mu_{i}^{1}\right\| ; \zeta \in \operatorname{cl} C\right\}=0 .
$$

Consequently,

$$
k \geqq \lim _{\varepsilon, \delta \rightarrow 0+} \sup _{\zeta \in C}\left\{2^{m} \pi^{m / 2}\left|d_{D}(\hat{\zeta})-\alpha\right|+v^{\varepsilon \delta}(\zeta)\right\},
$$

which completes the proof, because $k$ was an arbitrary number satisfying (66).

Combining lemmas 3.3 and 3.5 we obtain at once the following

3.6. Proposition. If $M \subset C$ is dense in $C$, then

$$
\omega W_{\alpha}=\lim _{\varepsilon, \delta \rightarrow 0+} \sup _{\zeta \in M}\left\{2^{m} \pi^{m / 2}\left|d_{D}(\hat{\zeta})-\alpha\right|+v^{\varepsilon \delta}(\zeta)\right\} .
$$

Remark. The above proposition (as well as its proof) is a complete analogue of 3.6 in [10].

The following lemma will enable us to derive a geometric expression for $\omega W_{\alpha}$.

3.7. Lemma. Define $v(r ; x)\left(r>0, x \in R^{m}\right)$ by (8) and let for $L \subset B, L \neq \emptyset$,

Then

$$
V_{0}(L)=\lim _{r \rightarrow 0+} \sup _{x \in L} v(r ; x) .
$$

$$
2^{m-1} \Gamma\left(\frac{1}{2} m\right) V_{0}(L)=\lim _{\varepsilon, \delta \rightarrow 0+} \sup \left\{v^{\varepsilon \delta}(\zeta) ; \zeta \in L \times\left\langle T_{1}, T_{2}\right)\right\},
$$

where $v^{\varepsilon \delta}(\zeta)$ has the meaning described in 3.3. 
Proof. Fix $L \subset B, L \neq \emptyset$. Note that $v^{\dot{\varepsilon} \delta}(\zeta)$ is a non-decreasing function of each of the variables $\varepsilon, \delta>0$ separately and

$$
\sup \left\{v^{\varepsilon \delta}(\xi, \tau) ; \tau \in\left\langle T_{1}, T_{2}\right)\right\}=v^{\varepsilon \delta}\left(\xi, T_{1}\right), \quad \xi \in B
$$

Hence

$$
\begin{aligned}
& \lim _{\varepsilon, \delta \rightarrow 0+} \sup \left\{v^{\varepsilon \delta}(\zeta) ; \zeta \in L \times\left\langle T_{1}, T_{2}\right)\right\}= \\
& \lim _{\varepsilon \rightarrow 0+} \sup \left\{v^{\varepsilon \delta}\left(\xi, T_{1}\right) ; \xi \in L, \delta=\frac{1}{4} \varepsilon^{4}\right\}
\end{aligned}
$$

Put

$$
r(\eta, \varepsilon)=\min \left(\varepsilon, \varepsilon^{2} \sqrt{ } \eta\right), \quad \eta>0
$$

and consider $\varepsilon, \delta$ satisfying

$$
0<\varepsilon<\left[4\left(T_{2}-T_{1}\right)\right]^{1 / 4}, \quad \delta=\frac{1}{4} \varepsilon^{4}
$$

Then

$$
v^{\varepsilon \delta}\left(\xi, T_{1}\right)=2^{m-1} \int_{0}^{\infty} \mathrm{e}^{-\eta} \eta^{m / 2-1} v(r(\eta, \varepsilon) ; \xi) \mathrm{d} \eta
$$

as it follows from (41), 2.2 and 1.8. Since

$$
v(r ; \xi) \leqq V<+\infty
$$

for any $\xi \in B$ and $r>0$, we get

$$
\lim _{\varepsilon \rightarrow 0+} \sup _{\xi \in L} \int_{\varepsilon^{-2}}^{\infty} \mathrm{e}^{-\eta} \eta^{m / 2-1} v(r(\eta, \varepsilon) ; \xi) \mathrm{d} \eta=0 .
$$

Taking into account that $r(\eta, \varepsilon)=\varepsilon^{2} \sqrt{ } \eta$ for $0 \leqq \eta \leqq \varepsilon^{-2}$ we obtain

$$
\begin{aligned}
& \lim _{\varepsilon \rightarrow 0+} \sup _{\xi \in L} \int_{0}^{\infty} \mathrm{e}^{-\eta} \eta^{m / 2-1} v(r(\eta, \varepsilon) ; \xi) \mathrm{d} \eta= \\
= & \lim _{\varepsilon \rightarrow 0+} \sup _{\xi \in L} \int_{0}^{\infty} \mathrm{e}^{-\eta} \eta^{m / 2-1} v\left(\varepsilon^{2} \sqrt{ }(\eta) ; \xi\right) \mathrm{d} \eta .
\end{aligned}
$$

Note now that

$$
\begin{aligned}
& \eta \geqq \varepsilon^{2} \Rightarrow v\left(\varepsilon^{2} \sqrt{ }(\eta) ; \xi\right) \geqq v\left(\varepsilon^{3} ; \xi\right) \\
& \eta \leqq \varepsilon^{-2} \Rightarrow v\left(\varepsilon^{2} \sqrt{ }(\eta) ; \xi\right) \leqq v(\varepsilon ; \xi)
\end{aligned}
$$


Hence we conclude

$$
\begin{gathered}
V_{0}(L) \Gamma\left(\frac{1}{2} m\right)=\lim _{\varepsilon \rightarrow 0+} \sup _{\xi \in L} v\left(\varepsilon^{3} ; \xi\right) \int_{\varepsilon^{2}}^{\varepsilon^{-2}} \mathrm{e}^{-\eta} \eta^{m / 2-1} \mathrm{~d} \eta \leqq \\
\leqq \lim _{\varepsilon \rightarrow 0+} \sup _{\xi \in L} \int_{0}^{\varepsilon^{-2}} \mathrm{e}^{-\eta} \eta^{m / 2-1} v\left(\varepsilon^{2} \sqrt{ }(\eta) ; \xi\right) \mathrm{d} \eta \leqq \\
\leqq \lim _{\varepsilon \rightarrow 0+} \sup _{\xi \in L} v(\varepsilon ; \xi) \int_{0}^{\varepsilon^{-2}} \mathrm{e}^{-\eta} \eta^{m / 2-1} \mathrm{~d} \eta=V_{0}(L) \Gamma\left(\frac{1}{2} m\right) .
\end{gathered}
$$

We see that

$$
\lim _{\varepsilon \rightarrow 0+} \sup _{\xi \in L} \int_{0}^{\varepsilon^{-2}} \mathrm{e}^{-\eta} \eta^{m / 2-1} v\left(\varepsilon^{2} \sqrt{ }(\eta) ; \xi\right) \mathrm{d} \eta=V_{0}(L) \Gamma\left(\frac{1}{2} m\right),
$$

which together with (73), (72), (71) completes the proof.

Remark. It is useful to extend the definition of $V_{0}(L)$ letting $V_{0}(\emptyset)=0$. Now we are in position to evaluate $\omega W_{\alpha}$ as follows.

\subsection{Theorem. Put}

$$
B_{1}=B \cap\left\{x ; d_{D}(x)=1\right\}, \quad B_{2}=R^{m} \cap\left\{x ; d_{D}(x)=\frac{1}{2}\right\}
$$

and write, for the sake of brevity,

$$
\begin{gathered}
\omega_{\alpha}=\frac{\omega W_{\alpha}}{2^{m-1} \Gamma\left(\frac{1}{2} m\right)}, \\
A=2 \pi^{m / 2} / \Gamma\left(\frac{1}{2} m\right), \\
V_{i}=V_{0}\left(B_{i}\right), \quad i=1,2
\end{gathered}
$$

(see also 3.7). Let us distinguish the following three cases:

(i) $B_{1}=\emptyset$ or $V_{2} \geqq \frac{1}{2} A+V_{1}$,

(ii) $B_{2}=\emptyset$ or $V_{1} \geqq \frac{1}{2} A+V_{2}$,

(iii) $B_{1} \neq \emptyset \neq B_{2}$ and $\left|V_{1}-V_{2}\right| \leqq \frac{1}{2} A$.

Then

$$
\begin{aligned}
& \omega_{\alpha}=A\left|\alpha-\frac{1}{2}\right|+V_{2} \text { in the case (i), } \\
& \omega_{\alpha}=A|\alpha-1|+V_{1} \quad \text { in the case (ii), }
\end{aligned}
$$

while in the case (iii)

$$
\omega_{\alpha}=\frac{1}{4} A+\frac{1}{2}\left(V_{1}+V_{2}\right)+A\left|\alpha-\left[\frac{3}{4}+\left(V_{1}-V_{2}\right) / 2 A\right]\right| .
$$


Proof. If $x \in B \backslash B_{1}$, then $d_{D}(x)<1$ and each ball $\Omega_{r}(x)(r>0)$ meets $R^{m} \backslash D$ in a set of positive $m$-measure; since $D$ is open, also

$$
H_{m}\left(\Omega_{r}(x) \cap D\right)>0 .
$$

Hence it follows by the relative isoperimetric inequality for sets with finite perimeter (see Theorem (4.3) in [16] or isoperimetric inequalities for currents established in [4], §6) that

$$
H_{m-1}\left(\Omega_{r}(x) \cap \hat{B}\right)>0,
$$

where $\widehat{B} \subset B_{2}$ is the reduced boundary of $D$ as defined in 2.4. In particular, $x \in \operatorname{cl} B_{2}$. We have thus shown that $B_{1} \cup B_{2}$ is dense in $B$. Put $L_{i}=B_{i} \times\left\langle T_{1}, T_{2}\right), M=$ $=L_{1} \cup L_{2}$, so that $M$ is dense in $C$ and we obtain from 3.6 that

$$
\omega W_{\alpha}=\lim _{\varepsilon, \delta \rightarrow 0+} \sup _{\zeta \in M}\left\{2^{m} \pi^{m / 2}\left|d_{D}(\hat{\zeta})-\alpha\right|+v^{\varepsilon \delta}(\zeta)\right\} .
$$

If $B_{1}=\emptyset$ then $d_{D}(\hat{\zeta})=\frac{1}{2}$ for each $\zeta \in M=L_{2}$ and 3.7 yields

$$
\omega W_{\alpha}=2^{m} \pi^{m / 2}\left|\frac{1}{2}-\alpha\right|+2^{m-1} \Gamma\left(\frac{1}{2} m\right) V_{2},
$$

which is in accordance with (76). Similarly, $B_{2}=\emptyset$ implies that $d_{D}(\hat{\zeta})=1$ for each $\zeta \in M=L_{1}$, whence we conclude by 3.7

$$
\omega W_{\alpha}=2^{m} \pi^{m / 2}|1-\alpha|+2^{m-1} \Gamma\left(\frac{1}{2} m\right) V_{1},
$$

which is the formula occuring in (77). Consider now the case when $B_{1} \neq \emptyset \neq B_{2}$ and let

$$
m_{i}=\lim _{\varepsilon, \delta \rightarrow 0+} \sup _{\zeta \in L_{i}}\left\{2^{m} \pi^{m / 2}\left|d_{D}(\hat{\zeta})-\alpha\right|+v^{\varepsilon \delta}(\zeta)\right\},
$$

so that (79) implies

$$
\omega W_{\alpha}=\max \left\{m_{1}, m_{2}\right\} .
$$

Since $d_{D}(\hat{\zeta})=2^{1-i}$ for each $\zeta \in L_{i}(i=1,2)$, we have by 3.7

$$
m_{i}=2^{m} \pi^{m / 2}\left|2^{1-i}-\alpha\right|+2^{m-1} \Gamma\left(\frac{1}{2} m\right) V_{i},
$$

whence it follows after simple calculation that

$$
\omega_{\alpha}=\frac{\max \left\{m_{1}, m_{2}\right\}}{2^{m-1} \Gamma\left(\frac{1}{2} m\right)}
$$

attains the value given by (76), (77) and (78), according as

$$
V_{2} \geqq \frac{1}{2} A+V_{1}, \quad V_{1} \geqq \frac{1}{2} A+V_{2} \text { and }\left|V_{1}-V_{2}\right| \leqq \frac{1}{2} A,
$$

respectively. This completes the proof. 
Remark. It would be interesting and useful to evaluate $\omega W_{\alpha}^{n}$, where $W_{\alpha}^{n}$ is the $n$-th power of the operator $W_{\alpha}$ (see [25], chap. X).

In connection with the operator

$$
H=\left(2^{m} \pi^{m / 2} \alpha I+W_{\alpha}\right)^{\prime}
$$

it is important to investigate

$$
g(\alpha)=\frac{\omega W_{\alpha}}{2^{m} \pi^{m / 2}|\alpha|}=\frac{\omega_{\alpha}}{A|\alpha|}, \quad \alpha \neq 0,
$$

and evaluate

$$
a=\inf \{g(\alpha) ; \alpha \neq 0\} .
$$

Indeed, the condition $a<1$ permits to apply the Riesz-Schauder theory to $H$. The above theorem enables us to establish the following corollary.

3.9. Theorem. Define a by (81), (80), A by (74) and $V_{i}$ by (75). Then

$$
a<1
$$

holds if and only if

$$
V_{1}<A \text { and } V_{2}<\frac{1}{2} A
$$

If the conditions (83) are fulfilled then

$$
g(\gamma)=a
$$

determines $\gamma$ uniquely and one of the following three cases must occur:

(i*) $B_{1}=\emptyset$,

(ii) $B_{2}=\emptyset$ or $V_{1} \geqq \frac{1}{2} A+V_{2}$,

(iii) $B_{1} \neq \emptyset \neq B_{2}$ and $\left|V_{1}-V_{2}\right| \leqq \frac{1}{2} A$.

The corresponding values of $a$ and $\gamma$ are then given as follows:

$$
\begin{aligned}
& A a=2 V_{2}, \quad \gamma=\frac{1}{2} \quad \text { in the case (i*), } \\
& A a=V_{1}, \quad \gamma=1 \quad \text { in the case (ii), }
\end{aligned}
$$

while in the case (iii)

$$
a=\frac{V_{1}+V_{2}+\frac{1}{2} A}{V_{1}-V_{2}+\frac{3}{2} A}, \quad \gamma=\frac{3}{4}+\frac{V_{1}-V_{2}}{2 A} .
$$


Proof. We shall distinguish the cases (i)-(iii) occuring in 3.8. Consider first the case (i). According to 3.8 we have in this case

$$
\begin{aligned}
& A g(\alpha)=\frac{\omega_{\alpha}}{|\alpha|}=\frac{A+V_{2}}{\alpha}-A . \text { for } 0<\alpha \leqq \frac{1}{2}, \\
& A g(\alpha)=A-\frac{\frac{1}{2} A+V_{2}}{\alpha} \text { for } \alpha<0, \\
& A g(\alpha)=A+\frac{V_{2}-\frac{1}{2} A}{\alpha} \text { for } \alpha \geqq \frac{1}{2} .
\end{aligned}
$$

Hence we see that $a<1$ implies $V_{2}<\frac{1}{2} A$, which together with (i) means that $B_{1}=\emptyset$ and $V_{1}=0$, so that (83), (i*) are fulfilled. Conversely, if (83) holds, then (85) $-(87)$ show that $a=2 V_{2} / A<1$ and $a$ is attained by $g$ at $\gamma=\frac{1}{2}$ only. Now we shall examine the case (ii). As it follows from 3.8,

$$
\begin{aligned}
& A g(\alpha)=\frac{V_{1}+A}{\alpha}-A \text { for } 0<\alpha \leqq 1, \\
& A g(\alpha)=A-\frac{A+V_{1}}{\alpha} \text { for } \alpha<0, \\
& A g(\alpha)=A+\frac{V_{1}-A}{\alpha} \text { for } \alpha \geqq 1
\end{aligned}
$$

We see that in this case (82) holds if and only if $V_{1}<A$, which is now just the same as (83). If (83) holds, then $a=V_{1} / A$ is attained by $g$ at $\gamma=1$ only. Finally, consider the case (iii). Employing 3.8 we get

$$
\begin{aligned}
& A g(\alpha)=\frac{V_{1}+A}{\alpha}-A \text { for } 0<\alpha \leqq \frac{V_{1}-V_{2}}{2 A}+\frac{3}{4}, \\
& A g(\alpha)=A-\frac{V_{1}+A}{\alpha} \text { for } \alpha<0, \\
& A g(\alpha)=A+\frac{V_{2}-\frac{1}{2} A}{\alpha} \text { for } \alpha \geqq \frac{V_{1}-V_{2}}{2 A}+\frac{3}{4} .
\end{aligned}
$$

Hence we conclude after simple calculation that $a<1$ if and only if $V_{2}<\frac{1}{2} A$, which is now equivalent with (83). If (83) holds, then $g$ attains its minimum at

$$
\gamma=\frac{3}{4}+\frac{V_{1}-V_{2}}{2 A}
$$


only and

$$
g(\gamma)=a=\frac{V_{1}+V_{2}+\frac{1}{2} A}{V_{1}-V_{2}+\frac{3}{2} A}
$$

The proof is complete.

Now it is easy to prove the following theorem concerning the second Fourier problem.

3.10. Theorem. Assume (83). Then for each $v \in \mathscr{B}^{\prime}$ there is a uniquely determined $\mu \in \mathscr{B}^{\prime}$ such that $H \mu=v$.

Proof. Fix $\gamma \in R^{1}$ satisfying (84) so that, by theorem 3.9,

$$
\frac{\omega W_{\gamma}}{2^{m} \pi^{m / 2}|\gamma|}=g(\gamma)<1 .
$$

Noting that

$$
H=\left(2^{m} \pi^{m / 2} \gamma I+W_{\gamma}\right)^{\prime}
$$

(see $2.8,2.7$ ) and writing $\beta=2^{m} \pi^{m / 2} \gamma$ we conclude by the Riesz-Schauder theory that

$$
\mathscr{B} \cap\left\{f ;\left(\beta I+W_{\gamma}\right) f=0\right\}=\mathscr{B}_{\gamma \beta}
$$

is finite dimensional and

$$
H\left(\mathscr{B}^{\prime}\right)=\mathscr{B}^{\prime} \cap\left\{v ;\left\langle\mathscr{B}_{\gamma \beta}, v\right\rangle=0\right\} .
$$

Since $\mathscr{B}_{\gamma \beta}$ is trivial by 2.9 , the same must hold of $\mathscr{B}^{\prime} \cap\{\mu ; H \mu=0\}$ and the proof is complete.

Remark. We see that weak characterization of the normal derivative by means of the functional $H$ permits application of potentialtheoretic methods to the Fourier problem for general domains satisfying (83). (Note that the boundary of such a domain need not be a hypersurface.) As it is well known, weak characterizations of boundary values occur frequently in the literature (see also [26], [10] for further references). We wish to note here that already J. Radon [21] referred to a related concept termed "Plemeljsche Randströmung" when treating the boundary value problems for logarithmic potentials in plane domains bounded by curves with bounded rotation. Unfortunately, the corresponding work of Plemelj [19] has not been available to the present author. Main results of this paper have been announced without proofs in [11].

Employing the integral representation derived in 2.5 one easily verifies that, for every $f \in \mathscr{B}, W f=u$ satisfies the equation

$$
\sum_{j=1}^{m} \partial_{j}^{2} u+\partial_{m+1} u=0
$$


in $R^{m+1} \backslash \mathrm{cl} C$. By duality based on the Riesz-Schauder theory we obtain thus the following result concerning the first problem of Fourier for the equation (88).

3.11. Theorem. Assume (83), define $D^{0}=R^{m} \cap\left\{x ; d_{D}(x)=0\right\}$ and put $E^{0}=$ $=D^{0} \times\left\langle T_{1}, T_{2}\right)$. Given $h \in \mathscr{B}$ there is an $f \in \mathscr{B}$ such that, for each $\zeta \in C \cap \mathrm{cl} E^{0}$,

$$
h(\zeta)=\lim W f(z), \quad z \rightarrow \zeta, \quad z \in E^{0} .
$$

If $B \subset \mathrm{cl} D^{\circ}$ then such an $f$ is uniquely determined.

Proof. Define $\gamma$ by (84) and put $\beta=2^{m} \pi^{m / 2} \gamma$. As we have seen in the proof of 3.10 , $\mathscr{B}_{\gamma \beta}$ is trivial. Hence we conclude that, given $h \in \mathscr{B}$, there is a uniquely determined $f \in \mathscr{B}$ such that

$$
\left(\beta I+W_{\gamma}\right) f=h .
$$

Now it is sufficient to employ theorem 2.6 (see also 2.7) showing that for $\zeta \in C \cap \mathrm{cl} E^{0}$

$$
\left(\beta I+W_{\gamma}\right) f(\zeta)=\lim W f(z), \quad z \rightarrow \zeta, \quad z \in E^{0} .
$$

Remark. It is easy to see that the assumption (47) introduced in the beginning of this chapter is a consequence of the weaker assumption

$$
V_{0}(B)<+\infty \text {. }
$$

Indeed, let us drop (47) and assume (89). Then there is an $r>0$ with

$$
\sup _{x \in B} v_{r}(x)<+\infty \text {. }
$$

Given $x \in B$ and $\varrho=\frac{1}{2} r$, there is a finite constant $K$ such that

$$
\int_{D \cap \Omega_{Q}(x)} \operatorname{div} v(x) \mathrm{d} x \leqq K
$$

for every vector-valued function $v=\left[v_{1}, \ldots, v_{m}\right]$ with infinitely differentiable components $v_{j}$ satisfying spt $v_{j} \subset \Omega_{\varrho}(x)(j=1, \ldots, m)$,

$$
\sum_{j=1}^{m} v_{j}^{2} \leqq 1
$$

This is obvious if $B \cap \Omega_{\varrho}(x)$ is contained in a hyperplane. In the opposite case one may fix points $x^{1}, \ldots, x^{m+1} \in B \cap \Omega_{\varrho}(x)$ that are not situated on a single hyperplane and employ the reasoning described in the proof of 2.10 in [10] to get a finite constant $K$ (depending on the quantities $v_{r}\left(x^{j}\right)$ and on mutual position of the points $x^{1}, \ldots, x^{m+1}$ ) such that (91) holds for all $v$ described above (note also that $\Omega_{\varrho}(x) \subset$ 
$\left.\subset \Omega_{r}\left(x^{j}\right), 1 \leqq j \leqq m+1\right)$. Since $B$ is compact, we conclude that $D$ has finite perimeter and

$$
\begin{aligned}
& v_{\infty}(x)=\int_{B} \frac{|n(y) \cdot(y-x)|}{|y-x|^{m}} \mathrm{~d} H_{m-1}(y), \\
& v_{r}(x)=\int_{B \cap \Omega_{r}(x)} \frac{|n(y) \cdot(y-x)|}{|y-x|^{m}} \mathrm{~d} H_{m-1}
\end{aligned}
$$

(see 2.12 and 2.8 in [10]). Now it is easy to see that (90) implies (47).

\section{References}

[1] E. De Giorgi: Su una teoria generale della misura $(r-1)$-dimensionale in uno spazio ad $r$ dimensioni, Ann. Mat. Pura Appl. (4) 36 (1954), 191-213.

[2] E. De Giorgi: Nuovi teoremi relativi alle misure $(r-1)$-dimensionali in uno spazio ad $r$ dimensioni, Ricerche di Matematica 4 (1955), 96-113.

[3] H. Federer: A note on the Gauss-Green theorem, Proc. Amer. Math. Soc. 9 (1958), 447-451.

[4] H. Federer and W. H. Fleming: Normal and integral currents, Ann. of Math. 72 (1960), $458-520$.

[5] G. Fichera: Alcuni recenti sviluppi della teoria dei problemi al contorno per le equazioni alle derivate parziali, Atti Convegno Equaz. Deriv. Parz. Trieste (1954), 174-227.

[6] W. H. Fleming: Functions whose partial derivatives are measures, Illinois J. Math. 4 (1960), $452-478$.

[7] A. Friedman: Partial differential equations of parabolic type, Prentice-Hall, 1964.

[8] L. I. Kamynin: Ljapunov's theorems for thermal potentials (Russian), Doklady ANSSSR 160 (1965), 271-273.

[9] J. Král: Note on sets whose characteristic functions have signed measures for their derivatives, Časopis pro pěstování matematiky 83 (1958), 116-221.

[10] J. Král: The Fredholm method in potential theory, Trans. Amer. Math. Soc. 125 (1966), $511-547$.

[11] J. Král: Flows of heat, Atti Accad. Naz. Lincei, Rend. Cl. Sc. fis., mat. e natur. 46 (1969), fasc. 2, 60-63.

[12] K. Krickeberg: Distributionen, Funktionen beschränkter Variation und Lebesguescher Inhalt nichtparametrischer Flächen, Ann. Mat. Pura Appl. (4) 44 (1957), 105-134.

[13] M. Krzyźański: Partial differential equations of the second order I (Polish), Warszawa 1957.

[14] O. A. Ladyženskaja, V. A. Solonnikov, N. N. Uralceva: Linear and quasilinear equations of - parabolic type (Russian), Moscow 1967.

[15] J. Mařik: The surface integral, Czechoslovak Math. J. (8I) 6 (1956), 522-558.

[16] M. Miranda: Distribuzioni aventi derivate misure, insiemi di perimetro localmente finito, Ann. Scuola Norm. Sup. Pisa (3) 18 (1964), 27-56.

[17] M. Pagni: Su un problema al contorno tipico per l'equazione del calore, Ann. Scuola Norm. Sup. Pisa (3) 11 (1957), 73-115.

[18] Chr. Y. Pauc: Functions with generalized gradients in the theory of cell functions. Ann. Math. Pura Appl. (4) 44 (1957), 135-152. 
[19] J. Plemelj: Potentialtheoretische Untersuchungen, Leipzig 1911.

[20] W. Pogorzelski: Sur la solution de l'équation intégrale dans le problème de Fourier, Ann. Soc. Polon. Math. 24 (1951), 56-74.

[21] J. Radon: Über die Randwertaufgaben beim logarithmischen Potential, Sitzungsber. Akad. Wiss. Wien (2a) 128 (1919), 1123-1167.

[22] F. Riesz, B. Sz. Nagy: Leçons d'analyse fonctionelle, Budapest 1952.

[23] L. Schwartz: Théorie des distributions I, II, Actualités Sci. Ind. 1091, 1122, Paris, 1950, 1951.

[24] Ju. D. Vurago and V.G. Maz'ja: Some questions in potential theory and function theory for regions with irregular boundaries (Russian), Zapiski naučn. sem. Leningrad. otd. MIAN 3 (1967).

[25] K. Yosida: Functional analysis, Springer 1965.

[26] L. C. Young: A theory of boundary values, Proc. London Math. Soc. (3) 14 A (1965), $300-314$.

Author's address: Praha 1, Žitná 25, ČSSR (Matematický ústav ČSAV v Praze). 